\title{
Impedance spectra of mixed conductors: a 2D study of ceria
}

\author{
Francesco Ciucci, ${ }^{a}$ Y Yong $\mathrm{Hao}^{b}$ and David G. Goodwin ${ }^{a}$ \\ Received 17th April 2009, Accepted 23rd September 2009 \\ First published as an Advance Article on the web 27th October 2009 \\ DOI: $10.1039 / \mathrm{b} 907740 \mathrm{e}$
}

\begin{abstract}
In this paper we develop an analytical framework for the study of electrochemical impedance of mixed ionic and electronic conductors (MIEC). The framework is based on non-equilibrium thermodynamics and it features the coupling of electrochemical reactions, surface transport and bulk transport processes. We utilize this work to analyze two-dimensional systems relevant for fuel cell science via the finite element method (FEM). Alternate current impedance spectroscopy (AC-IS or IS) of a ceria symmetric cell is simulated near equilibrium conditions (zero bias) for a wide array of working conditions including variations of temperature and $\mathrm{H}_{2}$ partial pressure on a two-dimensional doped ceria sample with patterned metal electrodes. The model shows agreement between computed IS curves and the experimental literature where the relative error on the impedance is consistently below $2 \%$. Important two-dimensional effects such as the impact of thickness decrease and the influence of variable electronic and ionic diffusivities on the impedance spectra are also explored.
\end{abstract}

\section{Introduction}

Mixed ionic and electronic conductors (MIEC), or in short mixed conductors, are substances capable of conducting both electrons and ions, and for that reason they are used in many applications, most notably in catalysis and eletrochemistry. They have been employed in gas sensors, fuel cells, oxygen permeation membranes, oxygen pumps and electrolyzers.

The study of the alternate current properties of MIEC aids the understanding of many of the physical chemical phenomena related to the behavior of defects, electrochemistry and interfaces. A technique frequently used to probe the interplay between these processes is impedance spectroscopy (IS). IS consists in injecting a "small" sinusoidal current into an electrochemical sample, a fuel cell for example, which is initially under steady state conditions. This perturbation in turn induces a small sinusoidal and dephased perturbation of the voltage. From the measurements of voltage and current over a wide set of frequencies, one can compute the complex impedance of the system. When the experiment is compared against a suitable model, impedance spectroscopy helps understand the linear physics of electroactive materials.

The tools used to deconvolve impedance spectra and relate them to physical chemical quantities are usually limited to one dimensional equivalent circuits. ${ }^{1,2}$ Even though the 1D approach is useful because it enables quick comparison of different physical chemical processes, it sometimes fails to help interpret satisfactorily physical chemical phenomena that extend to several dimensions. Only a handful of works attempted to scale up to two dimensions, and generally have been constrained to the steady-state setting. ${ }^{3-5}$

${ }^{a}$ California Institute of Technology, Department of Mechanical Engineering, Pasadena, CA 91125, USA.

E-mail:francesco@alumni.caltech.edu

${ }^{b}$ California Institute of Technology, Department of Materials Science, Pasadena, CA 91125,USA.E-mail: haoyong@caltech.edu
In this paper we develop a fast method for the computation of impedance spectra in the case of highly doped mixed conductors in 2D under geometrically symmetric conditions. The system studied was chosen so that it is not too cumbersome algebraically and readily relatable to experiments. However, the methodology is general and it can be easily extended to $3 \mathrm{D}$, to dissymmetric systems under non-zero bias and to complex chemical boundary conditions.

The paper proceeds as follows: we first determine the impedance equations ${ }^{6}$ and we develop a numerical framework for the solution of these spectroscopy equations, then we compare our simulation results with experimental data, and finally we study the influence of parameter variation on the IS: the thickness of the sample, the rates of the chemical reactions at the exposed MIEC surface and the diffusivity profiles.

More specifically for the mathematical portion of the paper: after nondimensionalization of the complete set of drift diffusion equations, we find that the ratio between the Debye length and the characteristic length scale of the material is remarkably large, hence we singularly perturb the governing equations and we deduce that electroneutrality is satisfied over the vast majority of the sample. Then we apply a small sinusoidal perturbation to the potential, which mathematically translates into a regular perturbation of the equations; after formal algebraic manipulations we collect first order terms and deduce two complex and linear partial differential equations in 2D space and time. Thanks to linearity, Fourier transformation of these equations and their boundary conditions leads to the determination of the complex impedance spectroscopy equations which we solve in $2 \mathrm{D}$ space for the frequencies of interest.

We verify our numerical results against experiments that are relevant for fuel cell applications. In particular, we study the case of a samaria doped ceria (SDC) sample, immersed in a uniform atmosphere of argon, hydrogen and water vapor. The sample is symmetric and reversible and has been the subject of 
extensive research. ${ }^{7-9}$ We find excellent agreement between the computed impedance spectra and experimental data. This shows that the approximations and the model are likely to be valid, hence this framework could help address a number of important fundamental physical/chemical issues in mixed conductors.

\section{System under study}

The physical system under study is a two-dimensional assembly which consists of a mixed oxygen ion and electron conductor slab of thickness $2 l_{2}$ sandwiched between two identical patterned metal current collectors, Fig. 1. The patterned collectors are repeated and symmetrical with respect to the center line $\Gamma_{1}$. Hence the system to be reduced to a repeating cell using the mirror symmetry lines $\Gamma_{1}, \Gamma_{2}$ and $\Gamma_{3}$. All sides of the sample are placed in a uniform gas environment. Two charge-carrying species are considered: oxygen vacancies, denoted by the subscript 'ion', and electrons, denoted by 'eon'.

The framework we propose is broad in scope; however, we narrow our study to samaria doped ceria. Doped ceria is a class of materials that has recently gained prominent relevance in fuel cell technology. ${ }^{10,11}$ We suppose that the uniform gas environment consists of a mixture of hydrogen and water vapor and we solve the electrochemical potential and current of both charge carriers using a linear and time-independent model, which we develop via perturbation techniques and Fourier transformation. We mainly compare our computational work to the data of Lai and Haile ${ }^{8}$ but we also leverage on some results of Chueh et al. ${ }^{7}$ to justify the boundary conditions. Both works study SDC-15 (15\% samarium doping), hence, the number of background dopant particles per unit volume, $B$, is well defined; it is assumed to be uniform and is reported in Table 1.

The surface dimensions are kept constant: the width of the metal/ceria interface $\left(\Gamma_{4}\right)$ is $2 W_{1}=3 \mu \mathrm{m}$ and the width of the gas $\mid$ ceria interface $\left(\Gamma_{5}\right)$ is $2 W_{2}=5 \mu \mathrm{m}$. The thickness of the MIEC is set to be $2 l_{2}=1 \mathrm{~mm}$, unless otherwise specified. Due to high electronic mobility in the metal, the thickness of

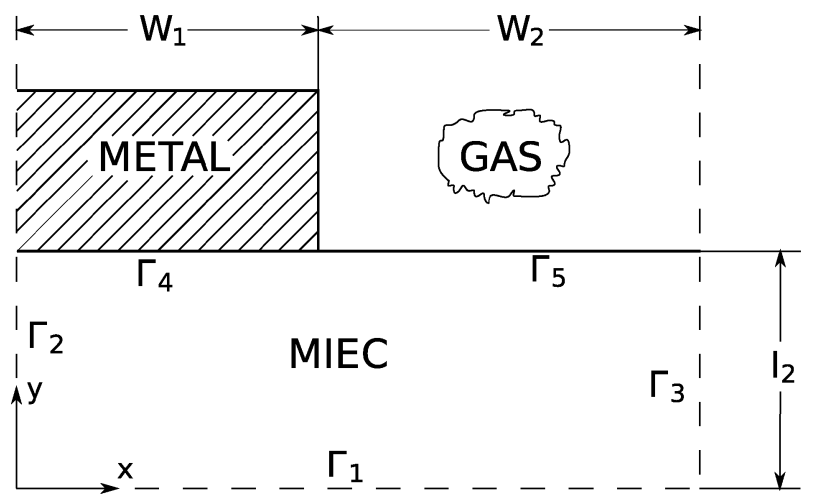

Fig. 1 Schematic depiction of the domain under study with annotation of the boundary names and dimensions. The domain is composed by an MIEC slab of half-thickness $l_{2}$ which is mirror symmetric with respect to $\Gamma_{1}$. On top of the slab there is a metal stripe infinitely long deposited over the surface $\Gamma_{4}$, the surface $\Gamma_{5}$ is exposed to the gas phase. The overall sample is mirror symmetric with respect to $\Gamma_{2}$ and $\Gamma_{3}$.
Table 1 Data for the domain geometry and background doping

\begin{tabular}{ll}
\hline$W_{1}$ & $1.5 \mu \mathrm{m}$ \\
$W_{2}$ & $2.5 \mu \mathrm{m}$ \\
$l_{2}$ & $500 \mu \mathrm{m}$ \\
$l_{c}$ & $10 \mu \mathrm{m}$ \\
$B$ & $3.47 \times 10^{27}$ particles per $\mathrm{m}^{3}$ \\
\hline
\end{tabular}

the metal stripe does not affect the calculation, and thus the thickness of the electrolyte is $l_{2}$, the thickness of the cell. Hence we assume that $l_{c}$, the characteristic length scale of the sample under study, is of the order of the sum of $W_{1}$ and $W_{2}$, giving $l_{c}=10 \mu \mathrm{m}$. The data mentioned above is summarized in Table 1.

The assumptions of the model are rather standard for MIEC. We assume that the vast majority of chemical reactions occur at the gas $\mid$ ceria interface ${ }^{12}$ or conversely that the gas $\mid$ metal|ceria interface, or triple-phase boundary (TPB), contributes little to the electrochemical reactions. We further treat the resulting surface chemistry as one global reaction, and do not consider diffusion of adsorbed species on the surface. ${ }^{13}$ Combined with the final assumption that the metal|ceria interface is reversible to electrons, i.e. an Ohmic condition, ${ }^{3}$ we are considering two steps in the electrode reaction pathway: surface reactions at the active site of the SDC $\mid$ gas interface and electron drift-diffusion from the active site to the metal current collector both along the SDC/gas interface and through the SDC bulk (Fig. 2).

We indicate the equilibrium quantities, such as electron and oxygen vacancy concentration, with the superscript (0). In order to determine equilibrium concentrations of charge carriers, we consider the following gas phase and bulk defect reactions:

$$
\begin{aligned}
\mathrm{H}_{2}(\mathrm{~g}) & +\mathrm{O}_{2}(\mathrm{~g}) \rightleftharpoons \mathrm{H}_{2} \mathrm{O}(\mathrm{g}) \\
\mathrm{O}_{\mathrm{O}}^{x} & \rightleftharpoons \mathrm{V}_{\mathrm{O}}^{\bullet \bullet}+\frac{1}{2} \mathrm{O}_{2}(\text { gas })+2 \mathrm{e}^{\prime}
\end{aligned}
$$

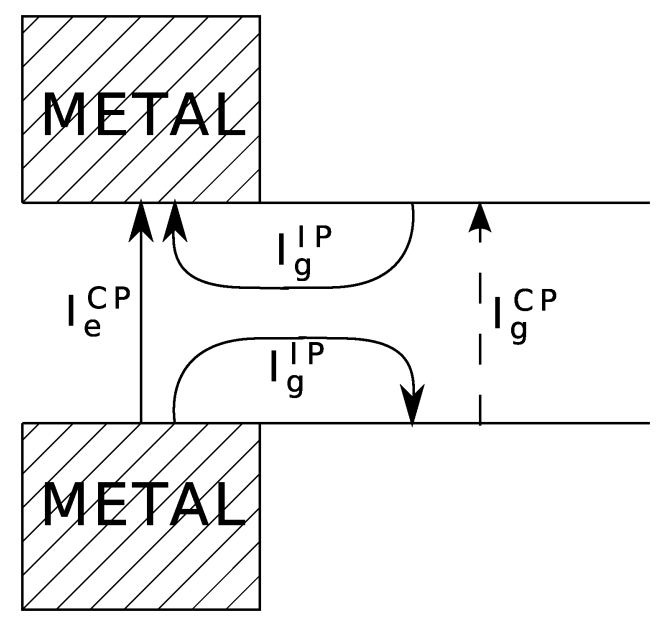

Fig. 2 Depiction of the currents in the MIEC. The superscript CP indicates cross-plane current and the superscript IP means in-plane currents. The subscript $g$ indicates that the flux is due to electrochemical reactions at the gas /ceria interface, while the subscript $e$ is for electrode to electrode current. We notice we will have four currents: one, the cross-plane electron flux $I_{e}^{\mathrm{CP}}$ from the bottom to the top electrode, two the cross-plane ionic flux from top to bottom gas|ceria interface $I_{g}^{\mathrm{CP}}$ and the in-plane electronic fluxes $I_{e}^{\mathrm{IP}}$ from the gas $\mid$ ceria interfaces to the electrodes. 
Table 2 Temperature range and material constants for the simulations

\begin{tabular}{lllll}
\hline$T$ & $500{ }^{\circ} \mathrm{C}$ & $550{ }^{\circ} \mathrm{C}$ & $600{ }^{\circ} \mathrm{C}$ & $650{ }^{\circ} \mathrm{C}$ \\
\hline$K_{g}$ & $5.059 \times 10^{+27}$ & $4.814 \times 10^{+25}$ & $7.757 \times 10^{+23}$ & $1.944 \times 10^{+22}$ \\
$K_{r}$ & $5.008 \times 10^{-22}$ & $2.263 \times 10^{-20}$ & $6.610 \times 10^{-19}$ & $1.340 \times 10^{-17}$ \\
$u_{\text {eon }} / \mathrm{m}^{2} \mathrm{~V}^{-2} \mathrm{~s}^{-1}$ & $4.762 \times 10^{-8}$ & $6.257 \times 10^{-8}$ & $6.873 \times 10^{-8}$ & $8.123 \times 10^{-8}$ \\
$u_{\text {ion }} / \mathrm{m}^{2} \mathrm{~V}^{-2} \mathrm{~s}^{-1}$ & $1.166 \times 10^{-9}$ & $2.070 \times 10^{-9}$ & $3.359 \times 10^{-9}$ & $4.936 \times 10^{-9}$ \\
\hline
\end{tabular}

where the Kröger-Vink notation is used, ${ }^{14}$ i.e., $\mathrm{V}_{\mathrm{O}}^{\bullet \bullet}$ is a vacant site in the crystal, $\mathrm{e}^{\prime}$ is an electron, and $\mathrm{O}_{\mathrm{O}}^{x}$ an oxygen site in the crystal (superscripts $\bullet, '$ and $x$ indicate respectively +1 charge, -1 charge and zero charge). At equilibrium the number of vacant sites per unit volume is $c_{\mathrm{ion}}^{(0)}$, and the number of electrons per unit volume is $c_{\mathrm{ion}}^{(0)}$. At equilibrium the following two quantities will be constants:

$$
\begin{aligned}
K_{g} & =\frac{\tilde{p}_{\mathrm{H}_{2} \mathrm{O}}^{2}}{\tilde{p}_{\mathrm{H}_{2}}^{2} \tilde{p}_{\mathrm{O}_{2}}^{2}}, \\
K_{r} & =\left(\frac{c_{\text {eon }}^{(0)}}{B}\right)^{2} \frac{c_{\mathrm{ion}}^{(0)}}{B} \tilde{p}_{\mathrm{O}_{2}}^{1 / 2},
\end{aligned}
$$

in addition to that, the electroneutrality condition is satisfied through the sample at equilibrium, giving

$$
1+\frac{c_{\mathrm{eon}}^{(0)}}{B}-2 \frac{c_{\mathrm{ion}}^{(0)}}{B}=0,
$$

where $\tilde{p}_{k}=\frac{p_{k}}{1 \text { atm }}$ and $p_{k}$ is the partial pressure of species $k$. In the dilute limit, at a given temperature and partial pressure, we solve for the equilibrium concentrations of vacancies $c_{\mathrm{ion}}^{(0)} \approx B / 2$ and electrons $c_{\mathrm{eon}}^{(0)} \approx B \frac{\sqrt{2 K r}}{\left[\mathrm{Sm}_{\mathrm{Ce}}\right]^{1.5} \tilde{p}_{\mathrm{O}_{2}}^{0.25}}$, where $\left[\mathrm{Sm}^{\prime}{ }_{\mathrm{Ce}}\right]$ is the concentration of dopant in the unit cell, in our case, $15 \%$. Finally we assume that the mobilities $u$ of all species are given in Table 2, from Lai and Haile. ${ }^{8}$

\section{Background}

\subsection{Asymptotic modeling of mixed conduction in the bulk}

A mixed conductor is a substance capable of conducting two or more charged species of opposite sign. Mass and charge transport in solids are described, at a mesoscopic level, by drift diffusion (DD) equations. The derivation of these equations is given in textbooks. ${ }^{15}$ For clarity we will shortly rewrite them here. For a mobile species $m$, the continuity portion of the DD equations is expressed by equations of the form

$$
\frac{\partial c_{m}}{\partial t}+\nabla \cdot \mathbf{j}_{m}^{P}=\dot{\omega}_{m}
$$

where $c_{m}$ is the concentration of species $m, \mathbf{j}_{m}^{P}$ is the particle (superscript $P$ ) flux of species $m$ per unit area and $\dot{\omega}_{m}$ is its net rate of creation per unit volume.

We will assume the following phenomenological relationship for the flux of species $m$ :

$$
\mathbf{j}_{m}^{P}=-\frac{c_{m} D_{m}}{k_{b} T} \nabla \tilde{\mu}_{m}
$$

where $D_{m}$ is its diffusivity, given by Einstein's relation $D_{m}=u_{m} k_{b} T / z_{m}$ ( $u_{m}$ is the mobility), and $\tilde{\mu}_{m}$ is its electrochemical potential, given by an expression of the type

$$
\tilde{\mu}_{m}=\mu_{m}^{0}+k_{b} T \log \left(c_{m} f_{m}\left(c_{m}, T, P\right)\right)+z_{m} e \phi .
$$

In the latter $e$ is the elementary charge, $\phi$ is the electric potential, $f_{m}$ is the activity of species $m$, and $z_{m}$ is its integer charge, i.e. -1 for electrons, +2 for oxygen vacancies in an oxide and $\mu_{m}^{0}$ is a reference value. We also define the $\star$-electrochemical potential of a species $m$ as

$$
\tilde{\mu}_{m}^{\star}=\frac{\tilde{\mu}_{m}}{z_{m}} .
$$

In this paper we suppose the presence of two mobile species: oxygen vacancies, which we indicate with the subscript 'ion' $\left(z_{\text {ion }}=+2\right)$, and electrons, subscript 'eon' $\left(z_{\text {eon }}=-1\right)$. The distribution of electrons and vacancies is thus described by 3 equations: one for the electric field (Poisson's equation for the potential) and two for the mobile species conservation. This set of equations can be written as

$$
\begin{gathered}
\Delta \phi=\frac{e}{\varepsilon}\left(B+c_{\text {eon }}-2 c_{\text {ion }}\right), \\
\partial_{t} c_{\text {eon }}+\nabla \cdot\left(-D_{\text {eon }} c_{\text {eon }} \nabla \frac{\tilde{\mu}_{\text {eon }}}{k_{b} T}\right)=0, \\
\partial_{t} c_{\text {ion }}+\nabla \cdot\left(-D_{\text {ion }} c_{\text {ion }} \nabla \frac{\tilde{\mu}_{\text {ion }}}{k_{b} T}\right)=0,
\end{gathered}
$$

where $\varepsilon$ is the permittivity of the medium, $B$ is the background dopant concentration in number of particles per unit volume and where we have chosen $\dot{\omega}_{\text {eon }}=\dot{\omega}_{\text {ion }}=0$. In the dilute limit, ${ }^{12,16-19}$ the chemical potential is described well using a Boltzmann distribution:

$$
\begin{aligned}
& \tilde{\mu}_{\text {eon }}=k_{b} T \log \left(\frac{c_{\text {eon }}}{c_{\text {eon }}^{0}}\right)-e \phi+\tilde{\mu}_{\text {eon }}^{0}, \\
& \tilde{\mu}_{\text {ion }}=k_{b} T \log \left(\frac{c_{\text {ion }}}{c_{\text {ion }}^{0}}\right)+2 e \phi+\tilde{\mu}_{\text {ion }}^{0},
\end{aligned}
$$

where $c_{\text {ion }}^{0}$ and $c_{\text {eon }}^{0}$ are reference values.

We have a sample under equilibrium conditions which we indicate with the superscript (0). We then perturb these equations with a small single harmonic excitation. The perturbation of the initial conditions is indicated with superscript (1). We then set all governing equations with their boundary conditions, in dimensionless form. Using singular asymptotic analysis gives that the electroneutrality condition is satisfied throughout most of the sample and the following set of equations are derived:

$$
\begin{array}{r}
i \omega \tau_{n}^{\star} \hat{n}^{(1)}-\Delta \hat{n}^{(1)}=0 \\
i \omega \tau_{\phi}^{\star} \hat{n}^{(1)}-\Delta \hat{\phi}^{(1)}=0
\end{array}
$$

Further details are given in Appendix A. 


\subsection{Boundary conditions}

The boundary conditions, even for this simple problem, are rather complex, we will first analyze the simple conditions that follow directly from the symmetry of the problem, subsequently we will approach the chemical boundary conditions. It follows from symmetry (Fig. 1) that $\partial_{\tilde{x}} \tilde{\phi}^{(1)}=$ $\partial_{\tilde{x}} n^{(1)}=0$ on $\Gamma_{2}$ and $\Gamma_{3}$. Since the metal is ion-blocking, $\frac{1}{2} \frac{\bar{n}}{\bar{p}} \partial_{\tilde{y}} n^{(1)}+2 \partial_{\tilde{y}} \tilde{\phi}^{(1)}=0$ will be satisfied on $\Gamma_{4}$. We have defined $\bar{n}=c_{\mathrm{eon}}^{(0)} / B$ and $\bar{p}=c_{\mathrm{eon}}^{(0)} / B$. We assume as well that the response of the metal to an electric perturbation is fast compared to the MIEC; from this it follows that we can take the electric potential $\tilde{\phi}^{(1)}$ uniform on $\Gamma_{4}$. Thanks to linearity and given the impedance setting, we can choose $\tilde{\phi}^{(1)}=$ $\frac{1}{\sqrt{2 \pi}} \Re\left(e^{i \omega \tau \tilde{t}}\right)$ on $\Gamma_{4}$ and $\tilde{\phi}^{(1)}=n^{(1)}=0$ on $\Gamma_{1}$.

We have assumed that the double layer's effects occurring on $\Gamma_{4}$ and $\Gamma_{5}$ are negligible. This assumption was motivated by two factors: firstly the experimental work of Lai and Haile ${ }^{9}$ was not able to capture any capacitive double layer effect; secondly we have assumed that $\Gamma_{4}$ is only weakly charged and that on $\Gamma_{5}$ electroneutrality is essentially satisfied due to the quickness of transfer. We can expect, however, that double layer effects are significant if the excitation frequency is sufficiently high. In this case, the nonpenetration condition for the electroneutral model breaks down and a capacitive effect needs to be added as shown by Fleig et al. ${ }^{20-22}$

We assume the chemistry of the reactions occurring at the interface between MIEC and gas, i.e., $\Gamma_{5}$, has a finite speed and that it is correctly characterized by a one step reaction. ${ }^{23}$ For simplicity we start from

$$
\mathrm{H}_{2}(\mathrm{~g}) \rightleftharpoons \mathrm{H}_{2} \mathrm{O}(\mathrm{g})+\mathrm{V}_{\mathrm{O}}^{\bullet \bullet}+2 \mathrm{e}^{\prime}
$$

We also remark ${ }^{23}$ that the rates of injection of vacancies $\dot{\omega}_{\text {ion, } S}$ and electrons $\dot{\omega}_{\text {eon }, S}$ at $\Gamma_{5}$ satisfy (subscript $S$ indicates surface) the following two equations:

$$
\begin{aligned}
& \dot{\omega}_{\mathrm{ion}, S}=k_{f} \tilde{p}_{\mathrm{H}_{2}}-k_{r} \tilde{p}_{\mathrm{H}_{2} \mathrm{O}} c_{\mathrm{ion}} c_{\mathrm{eon}}^{2} \\
& \dot{\omega}_{\mathrm{eon}, S}=2 \dot{\omega}_{\mathrm{ion}, S},
\end{aligned}
$$

where $k_{f}$ is the forward rate of the reaction in (11) and $k_{r}$ is the reverse rate.

The latter gives, under small perturbation assumptions, ${ }^{23} \mathrm{a}$ Chang-Jaffé boundary condition ${ }^{24}$

$$
-\dot{\omega}_{\mathrm{eon}, S}^{(1)}=4 \frac{D_{\mathrm{ion}}}{l_{c}} \tilde{k}_{f}^{0} \tilde{p}_{\mathrm{O}_{2}}^{1 / 4}\left(1+\frac{c_{\mathrm{eon}}^{(0)}}{4 c_{\mathrm{ion}}^{(0)}}\right) \tilde{p}_{\mathrm{H}_{2}} n^{(1)} .
$$

We define $k_{f}=2 \frac{D_{\text {ion }}}{l_{c}} \tilde{k}_{f}$ and $\tilde{k}_{f}=\tilde{k}_{f}^{0} \tilde{p}_{\mathrm{O}_{2}}^{\beta} \times \frac{\# \text { particles }}{m^{3}}, \dagger$ where we choose $\beta=1 / 4 .^{23} \mathrm{~A}$ further comment is necessary; the electrochemical oxidation of $\mathrm{H}_{2}$ at the ceria|gas interface is not well understood at atomistic or even at kinetic level. However, it is clear that, as already outlined elsewhere, ${ }^{23}$ the expression (13) follows directly from linearization and subsequent projection of a complex electrochemical mechanism. As shown in work by Ciucci et al., ${ }^{23}$ unless we

$\dagger$ The order of magnitude of the fitted injection rate $k_{f}$ can be easily computed. If we take $p_{\mathrm{O}_{2}}=10^{-24}, l_{c}=10^{-5} \mathrm{~m}, D_{\text {ion }}=10^{-10} \mathrm{~m}^{2} \mathrm{~s}^{-1}$ and $\tilde{k}_{f}^{0} \approx 10^{32}$, we will have $k_{f} \approx 10^{32} \times \frac{10^{-10}}{10^{-5}} \times 10^{-6}=$ $10^{21} \frac{\# \text { particles }}{\mathrm{m}^{2}} \approx 10^{-3} \frac{\mathrm{mol}}{\mathrm{m}^{2}}=10^{-7} \frac{\mathrm{mol}}{\mathrm{cm}^{2}}$. assume $-\dot{\omega}_{\mathrm{eon}, S}^{(1)}$ having $\beta=1 / 4$, the experimental data of the polarization resistance cannot be reproduced.

Hence the $y$-flux of electrons and vacancies satisfies the following expression along $\Gamma_{5}: \mathbf{j}_{\mathrm{eon}}^{P} \cdot \mathbf{e}_{y}=2 \mathbf{j}_{\mathrm{ion}}^{P} \cdot \mathbf{e}_{y}=-\dot{\omega}_{\mathrm{eon}, S}$. If we define $\quad \tilde{A}_{\phi}=\tilde{k}_{f} \frac{\tilde{p}_{\mathrm{H}_{2}}}{c_{\text {ion }}^{(0)}}\left(1-\frac{D_{\text {ion }}}{D_{\text {eon }}}\right) \quad$ and $\tilde{A}_{n}=\tilde{k}_{f} \frac{\tilde{p}_{\mathrm{H}_{2}}}{c_{\mathrm{ion}}^{(0)}}\left(1+4 \frac{D_{\text {ion }} c_{\text {ion }}^{(0)}}{D_{\text {eon }} c_{\text {oon }}^{(0)}}\right)$, we can rewrite the boundary conditions on $\Gamma_{5}$ as $\partial_{\tilde{y}} \tilde{\phi}^{(1)}=\tilde{A}_{\phi} n^{(1)}$ and $\partial_{\tilde{y}} n^{(1)}=\tilde{A}_{n} n^{(1)}$. In Fourier space the boundary conditions can be summarized as follows:

$$
\begin{cases}\hat{\phi}^{(1)}=0 \& \hat{n}^{(1)}=0 & \text { on } \Gamma_{1} \\ \partial_{\tilde{x}} \hat{\phi}^{(1)}=0 \& \partial_{\tilde{x}} \hat{n}^{(1)}=0 & \text { on } \Gamma_{2} \& \Gamma_{3} \\ \hat{\phi}^{(1)}=1 \& \partial_{\tilde{y}} \hat{n}^{(1)}=-4 \frac{\bar{p}}{\bar{n}} \partial_{\tilde{y}} \hat{\phi}^{(1)} & \text { on } \Gamma_{4} \\ \partial_{\tilde{y}} \hat{\phi}^{(1)}=\tilde{A}_{\phi} \hat{n}^{(1)} \& \partial_{\tilde{y}} \hat{n}^{(1)}=\tilde{A}_{n} n^{(1)} & \text { on } \Gamma_{5}\end{cases}
$$

\subsection{Numerical solution procedure for the $2 \mathrm{D}$ case}

In order to solve numerically the equations (43) with boundary conditions (44) (see Appendix A) we employ an h-adapted finite element method (FEM), implemented with FreeFem $++{ }^{25}$ The governing equations are discretized on a triangular unstructured mesh using quadratic continuous basis functions with a centered third order bubble. We use a direct method to solve the linear system following integration of (43) in the discretized mesh. Then the mesh is adaptively refined nine times for each case. The a posteriori adaptation is performed the first six times against the 4 -dimensional vector $\left(\nabla \Re\left[\hat{\mu}_{\text {eon }}^{(1)}\right], \nabla \Re\left[\hat{\mu}_{\text {ion }}^{(1)}\right]\right)$ and subsequently against $\eta_{\varepsilon}$ (see Appendix A). The h-adaptation ensures high regularity of the $\mathrm{H}^{1}$ a posteriori estimator, ${ }^{26}$ locally below $10^{-5}$, and it guarantees that the mesh is finer where sharper gradients occur. Independently of frequency, mesh adaptivity results in coarseness everywhere except in the vicinity of the interfaces - in particular the refinement increases towards the triple-phase boundary (the intersection of metal, oxide and gas phases, which is though to be a particularly active site for electrochemical reactions ${ }^{11,27}$ ); this fact indicates strong nonlinearities around that area. Finally we note that FreeFem ++ execution time is comparable to custom-written $\mathrm{C}++$ code and its speed is enhanced by the utilization of fast sparse linear solvers such as the multi-frontal package UMFPACK ${ }^{28}$ Due to the sparsity of the problem we make extensive use of this last feature.

We further note that the utilization of asymptotic expansion and Fourier transformation techniques, while guaranteeing linearity, has a significant speed advantage over direct sinusoidal $^{29}$ and step relaxation techniques ${ }^{30}$ applied to nonlinear problems. This method can be directly used to examine chemical reactions within the cell and draw direct conclusions about fast and rate-limiting chemical reactions. Also, this procedure lends itself to systematic error estimation and its implementation can be done automatically for a time-dependent problem. ${ }^{31}$

\subsection{The 1D case: an analytical solution}

Since we also aim to compare the $1 \mathrm{D}$ and $2 \mathrm{D}$ solutions, it is beneficial to revisit the solution of the $1 \mathrm{D}$ version of eqn (10). ${ }^{6}$ 
Table 3 Definitions of the terms in the 1D model

\begin{tabular}{ll}
\hline$R_{\text {ion }}^{\perp}$ & Measured \\
\hline$R_{\text {eon }}$ & $2 l_{2} / \sigma_{\text {eon }}$ \\
$R_{\text {ion }}$ & $2 l_{2} / \sigma_{\text {ion }}$ \\
$R_{0}$ & $1 /\left(1 / R_{\text {eon }}+1 /\left(R_{\text {ion }}+2 R_{\text {ion }}^{\perp}\right)\right)$ \\
$R_{\infty}$ & $1 /\left(1 / R_{\text {eon }}+1 / R_{\text {ion }}\right)$ \\
$C_{\text {chem }}$ & $\frac{e^{2}}{k_{b} T} 2 l_{2} /\left(1 /\left(z_{\text {eon }}^{2} c_{\text {eon }}\right)+1 /\left(z_{\text {ion }}^{2} c_{\text {ion }}^{(0)}\right)\right)$ \\
$\tilde{D}$ & $4 l_{2}^{2} /\left(\left(R_{\text {ion }}+R_{\text {eon }}\right) C_{\text {chem }}\right)$ \\
$s$ & $\sqrt{i 4 \omega l_{2}^{2} /(4 \tilde{D})}$ \\
\hline
\end{tabular}

It is easy to show that the solution $\left(\hat{n}^{(1)}, \hat{\phi}^{(1)}\right)$ will be (if $\omega \neq 0$ ) such that:

$$
\begin{aligned}
& \hat{n}^{(1)}=\sum_{ \pm} a_{ \pm} e^{ \pm \sqrt{i} \sqrt{\tau_{n}^{\star} \omega} \tilde{y}}, \\
& \hat{\phi}^{(1)}=\hat{\phi}_{0}^{(1)}+\left(\hat{\phi}_{0}^{(1)}\right)^{\prime} \tilde{y}+\frac{\tau_{\phi}^{\star}}{\tau_{n}^{\star}} \hat{n}^{(1)},
\end{aligned}
$$

where for simplicity we indicate $\sqrt{i}=e^{i \frac{\pi}{4}}$, and where $a_{ \pm}, \hat{\phi}_{0}^{(1)}$ and $\left(\hat{\phi}_{0}^{(1)}\right)^{\prime}$ are constants. The boundary conditions, as in the $2 \mathrm{D}$ case, at $\tilde{y}=0\left(\Gamma_{1}\right)$ are

$$
\hat{\phi}^{(1)}=0 \text { and } \hat{n}^{(1)}=0 .
$$

The latter can help rewrite equations (15) as

$$
\begin{aligned}
& \hat{n}^{(1)}=2 a_{+} \sinh \left(\sqrt{i} \sqrt{\tau_{n}^{\star} \omega} \tilde{y}\right), \\
& \hat{\phi}^{(1)}=\left(\hat{\phi}_{0}^{(1)}\right)^{\prime} \tilde{y}+2 a_{+} \frac{\tau_{\phi}^{\star}}{\tau_{n}^{\star}} \sinh \left(\sqrt{i} \sqrt{\tau_{n}^{\star} \omega} \tilde{y}\right) .
\end{aligned}
$$

If we set $\gamma_{\phi}=\frac{R_{\text {ion }}^{\perp} e l_{c} D_{e} c_{\text {eon }}^{(0)}}{U_{T}\left(1+\frac{1}{4} \overline{\bar{p}}\right)}$ and $\gamma_{n}=\frac{1}{4} \frac{\bar{n}}{\bar{p}} \gamma_{\phi}$, then at $\tilde{y}=l_{2}$ we have the following two conditions: ${ }^{9}$

$$
\hat{\phi}^{(1)}=1 \& \hat{n}^{(1)}+\gamma_{\phi} \frac{\mathrm{d} \hat{\phi}^{(1)}}{\mathrm{d} \tilde{y}}+\gamma_{n} \frac{\mathrm{d} \hat{n}^{(1)}}{\mathrm{d} \tilde{y}}=0 .
$$

The boundary conditions (18) will lead to the determination of $a_{+}$and $\left(\hat{\phi}_{0}^{(1)}\right)^{\prime}$ in (17) and the 1D model leads to impedance of the form: ${ }^{8,32,33}$

$$
\begin{aligned}
Z_{1 D}\left(\omega, \tilde{p}_{\mathrm{O}_{2}}, T\right)= & R_{\infty}+\left(R_{0}-R_{\infty}\right)\left(1+\frac{R_{\text {ion }}+R_{\text {eon }}}{2 R_{\text {ion }}}\right) \\
& \times \frac{\tanh s}{s+\frac{R_{\text {ion }}+R_{\text {eon }}}{2 R_{\text {ion }}^{\perp}} \tanh s},
\end{aligned}
$$

where all the relevant terms are reported in Table 3 . In the case of a ceria under reducing conditions such impedance has a tear-drop shaped Nyquist plot.

\section{Results}

\subsection{Comparison with experiments}

The electron electrochemical potential drop across the sample, i.e. the electron electrochemical potential difference between the top and bottom electrodes $\left(\Gamma_{4}\right.$ and its symmetric reflection), is given by the following expression:

$$
\hat{V}^{(1)}=2 U_{T}\left[\left\langle\left(\hat{\mu}_{e}^{(1)}\right)^{\star}\right\rangle_{\Gamma_{4}}-\left\langle\left(\hat{\mu}_{e}^{(1)}\right)^{\star}\right\rangle_{\Gamma_{1}}\right],
$$

where $\langle a\rangle_{\Gamma}$ indicates the average of the quantity $a$ over the set $\Gamma$. At first order the $\star$-electrochemical potential is given by $\left(\hat{\mu}_{e}^{(1)}\right)^{\star}=\hat{\phi}^{(1)}-\hat{n}^{(1)}$. The electric current density at the two ends of the circuit is

$$
\hat{j}^{(1)}=\frac{D_{\mathrm{eon}} e c_{\mathrm{eon}}^{(0)} \int_{\Gamma_{4}} \nabla_{\tilde{x}}^{\tilde{\mu}_{\mathrm{en}}^{(1)}} \cdot \mathbf{e}_{y} \mathrm{~d} \tilde{x}}{\left(W_{1}+W_{2}\right) l_{c}} .
$$

Hence, the 2D impedance is given by the expression

$$
Z_{2 D}\left(\omega, \tilde{p}_{\mathrm{O}_{2}}, T\right)=\hat{V}^{(1)} / \hat{j}^{(1)} .
$$

We define the error of the $2 \mathrm{D}$ impedance $Z_{2 D}$ with respect to experimental impedance $Z_{1 D}$ spectra (19) as follows:

$$
\varepsilon_{F}\left(\omega, \hat{p}_{\mathrm{O}_{2}}, T\right)=\left|1-\frac{Z_{2 D}\left(\omega, \hat{p}_{\mathrm{O}_{2}}, T\right)}{Z_{1 D}\left(\omega, \hat{p}_{\mathrm{O}_{2}}, T\right)}\right| .
$$

For every data point, uniquely defined by the couple $\left(\hat{p}_{\mathrm{O}_{2}}, T\right)$, we fit the $2 \mathrm{D}$ data against the measured $1 \mathrm{D}$ equivalent circuit data ${ }^{9}$ by minimizing $\varepsilon_{F}\left(\omega, \hat{p}_{\mathrm{O}_{2}}, T\right)$ with respect to the surface reaction constant $\tilde{k}_{f}^{0}=A \hat{p}_{\mathrm{O}_{2}}^{\alpha}$, which is a function of both $\mathrm{O}_{2}$ partial pressure and temperature. We remark that $\tilde{k}_{f}^{0}$ is the sole parameter we allowed to vary in this procedure and all other data was obtained from the literature and presented in Tables 1 and 2 . With only one parameter variation, we obtained excellent agreement between experimental results and $2 \mathrm{D}$ calculations, i.e., $\varepsilon_{F}\left(\omega, \hat{p}_{\mathrm{O}_{2}}, T\right)<2 \%$. As an example, $2 \mathrm{D}$ impedance results at four different oxygen partial pressures and at $650{ }^{\circ} \mathrm{C}$ are shown in Fig. 3. The IS calculation shows, in accordance with experimental results, that the resulting arc is tear drop shaped as shown for ceria using a transmission line type of model as in section 3.4. Similar results are recovered if the diffusivities are no longer uniform. We computed the $\tilde{k}_{f}^{0}$ by minimizing the $\varepsilon_{F}$ for a total of 28 cases ( 7 pressures times 4 temperature). We report in Table 4 the results of linear regression of these minimizing values (each line is derived by keeping the temperature fixed and varying $\hat{p}_{\mathrm{O}_{2}}$ ). We also write in Table 4 , the $95 \%$ confidence intervals for the fitting of $A$, i.e. $A \approx \bar{A} \pm \varepsilon_{A}$, and $\alpha$, i.e. $\alpha=\bar{\alpha} \pm \varepsilon_{\alpha}$; we finally report the root mean square error $\sigma$ and the adjusted $R^{2}$ value ${ }^{34}$ (where a value close to unity indicates a perfect fit while negative values indicate poor data correlation). Directly from the analysis of Table 4 we deduce that $\tilde{k}_{f}^{0}$ fitting to a straight line is reasonable for "high" temperatures $\left(T \geq 550{ }^{\circ} \mathrm{C}\right)$. We note that $\tilde{k}_{f}^{0}$ is temperature-dependent via $\bar{A}(\bar{A}$ decreases with $T)$. Furthermore $\tilde{k}_{f}^{0}$ is slightly pressure dependent via the coefficient $\alpha$, the average value of $\bar{\alpha} \approx 0.05 \geq 0$; however, the error is of the same order of the slope. Hence the total rate of reaction is likely to be $\dot{\omega}_{\text {eon }, S} \propto \tilde{p}_{\mathrm{O}_{2}}^{-1 / 4+\beta_{0}}$ where $\alpha_{0}$ lies somewhere in the set $[0,0.1]$.

\subsection{The polarization resistance in frequency space}

One of the goals of fuel cell science is to understand and possibly reduce the polarization resistance, i.e. that portion of 

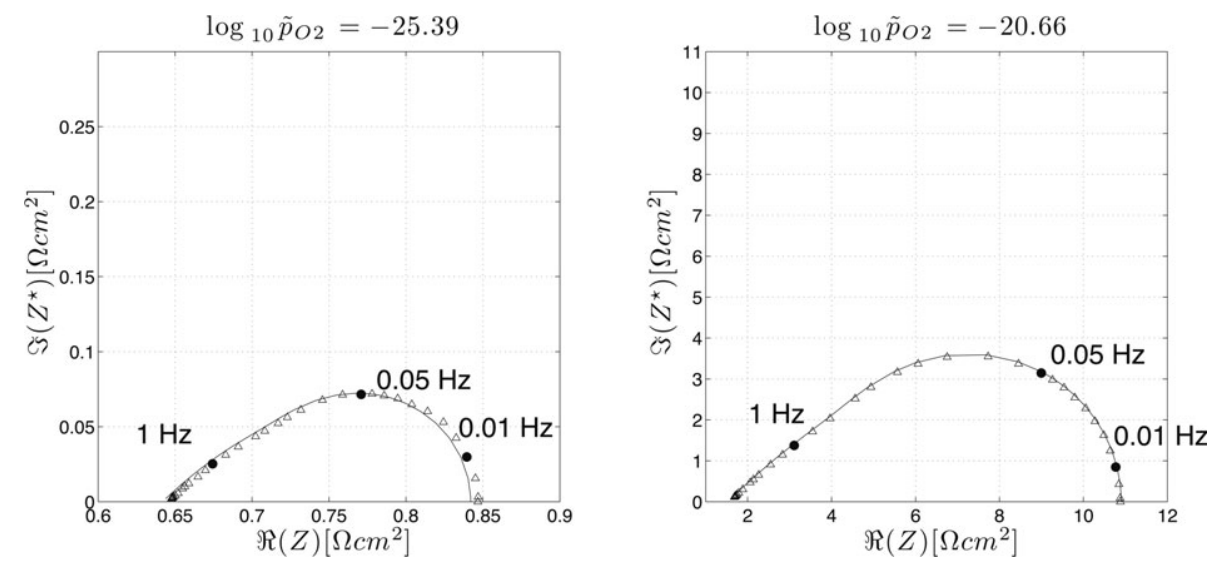

Fig. $3 \mathrm{AC}$ impedance plot of the system depicted in Fig. 1. The results are presented at $650{ }^{\circ} \mathrm{C}$ at two different oxygen partial pressures $\tilde{p}_{\mathrm{O}_{2}}$. The triangles indicate experimental values while the solid line is computed using the $2 \mathrm{D}$ framework.

Table 4 Fitted values of $\tilde{k}_{f}^{0}=A \tilde{p}_{\mathrm{O}_{2}}^{\alpha}, 95 \%$ confidence interval

\begin{tabular}{lllllrl}
\hline$T /{ }^{\circ} \mathrm{C}$ & $\log _{10} \bar{A}$ & $\log _{10} \varepsilon_{A}$ & $\bar{\alpha}$ & $\varepsilon_{\alpha}$ & \multicolumn{1}{l}{$R^{2}$} & \multicolumn{1}{l}{$\sigma$} \\
\hline 500 & 32.48 & 0.150 & 0.05349 & 0.1655 & -0.0439 & 0.1577 \\
550 & 32.10 & 0.045 & 0.04160 & 0.0482 & 0.7622 & 0.04589 \\
600 & 32.02 & 0.055 & 0.06674 & 0.0637 & 0.5378 & 0.06067 \\
650 & 31.95 & 0.055 & 0.05596 & 0.0623 & 0.4981 & 0.05938 \\
\hline
\end{tabular}

the resistance due to electric field effects at interfaces. For that purpose it is key to identify and understand the main processes that define this quantity. Specifically, the area specific polarization resistance for our system is defined as ${ }^{23}$

$$
Z_{\text {ion }}^{\perp}=U_{T} \frac{\left\langle\hat{\mu}_{\text {ion }}^{\star}\right\rangle_{\Gamma_{5}}-\left\langle\hat{\mu}_{\text {en }}^{\star}\right\rangle_{\Gamma_{4}}}{\hat{j}_{\mathrm{IP}}^{(1)}},
$$

where $\hat{j}_{\mathrm{IP}}^{(1)}=\frac{1}{W_{1}+W_{2}} \int_{\Gamma_{5}} \dot{\omega}_{\mathrm{eon}, S} \mathrm{~d} x$ is the ionic contribution to the area specific current (Fig. 2). The $Z_{\text {ion }}^{\perp}$ can be understood as the sum of a surface resistance $Z_{\text {surf }}$ and a bulk polarization resistance, $Z_{\text {bulk }}=Z_{\text {ion }}^{\perp}-Z_{\text {surf }}$, where the $Z_{\text {surf }}$ is the portion of the area specific resistance due to effects of the exposed boundary $\Gamma_{5}$ and it is given by

$$
Z_{\text {surf }}=U_{T} \frac{\left\langle\hat{\mu}_{\text {ion }}^{\star}\right\rangle_{\Gamma_{5}}-\left\langle\hat{\mu}_{\text {eon }}^{\star}\right\rangle_{\Gamma_{5}}}{\hat{j}_{\mathrm{IP}}^{(1)}} .
$$

In our model, by definition, the $Z_{\text {surf }} \in \mathbb{R}^{+}$is proportional to $\left(1+W_{1} / W_{2}\right)$ and inversely proportional to both $\tilde{p}_{\mathrm{H}_{2}}$ and $k_{f}$

$$
Z_{\text {surf }}=\frac{1}{2}\left(1+\frac{W_{1}}{W_{2}}\right) \frac{U_{T}}{e k_{f} \tilde{p}_{\mathrm{H}_{2}}} .
$$

The fraction $f_{\text {surf }}=\frac{Z_{\text {surf }}}{Z^{\perp}}$ indicates what portion of the polarization impedance is due to surface effects. From Fig. 4 we note two fundamental facts: first, as we expect, at "lower" injection rates $f_{\text {surf }}$ increases - physically this means that if the chemistry is sufficiently slow it will dominate the polarization resistance leading to an $f_{\text {surf }}$ of approximately unity. Second, we notice some frequency dependent behavior of $R_{\text {ion }}^{\perp}$. Our computations show that $f_{\text {surf }}$ decreases with $\omega$ while the dephasing between $Z_{\text {surf }}$ and $Z_{\text {ion }}^{\perp}$, described by $\arg \left(f_{\text {surf }}\right)$, increases with $\tilde{k}_{f}^{0}$ and decreases with $\omega$. The
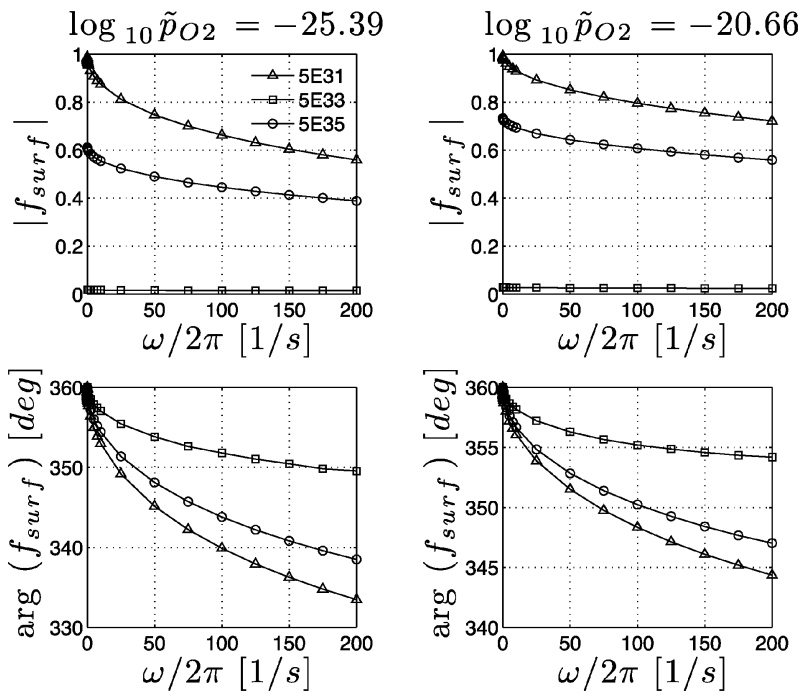

Fig. 4 Plot of $f_{\text {surf }}=\frac{R_{\text {surf }}}{R_{\text {t. }}}$ as a complex valued function of frequency $f=\omega / 2 \pi$. We present two cases, both at $650{ }^{\circ} \mathrm{C}$, the one to the left at reducing conditions $\tilde{p}_{\mathrm{O}_{2}}=10^{-25.33}$ and the one to the right at $\tilde{p}_{\mathrm{O}_{2}}=10^{-20.66}$, parametrized versus ${\tilde{k_{f}^{(0)}}}^{(0)} 5 \times 10^{31}, 5 \times 10^{32}, 5 \times 10^{33}$.

behavior of $f_{\text {surf }}$ in phase space clearly shows that $Z_{\text {surf }}$ includes two interrelated processes:

1. reactions on the surface exposed to the gas;

2. transport of charged species in MIEC.

Within this framework, as $\omega$ increases, the losses in the polarization due to drift diffusion increase and surpass the (constant) reaction or surface losses.

\subsection{Analysis of the $2 \mathrm{D}$ solution}

4.3.1 Qualitative considerations. We can use the framework developed here to study the Fourier transform of the electrochemical potentials of electrons and vacancies $\hat{\mu}_{\text {eon }}^{(1)}=$ $\hat{n}^{(1)}-\hat{\phi}^{(1)}$ and $\hat{\mu}_{\text {ion }}^{(1)}=\hat{\phi}^{(1)}+\frac{\bar{n}}{2 \bar{p}} \hat{n}^{(1)}$ as functions of frequency. In Fig. 5 and 6 we plot the 2D distributions of the dimensionless electrochemical potential in the computational domain at $T=650{ }^{\circ} \mathrm{C}, \tilde{p}_{\mathrm{O}_{2}}=10^{-25.33}$ and $\tilde{k}_{f}^{0}=10^{32}$ with frequency $\omega$ increasing from $10^{-3}$ to $10^{5} \mathrm{rad} \mathrm{s}^{-1}$. Thanks to the Fig. 5 and 6 , we can address the qualitative behavior of the solution. 

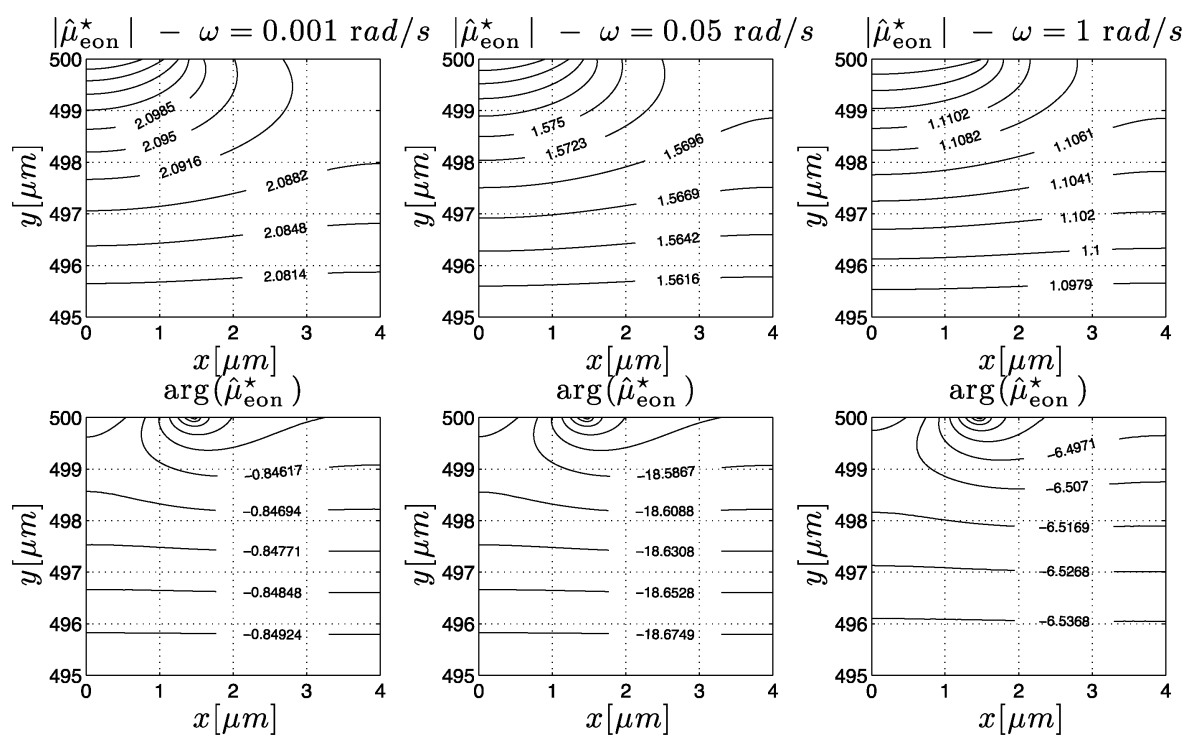

Fig. 5 Plots of the complex electrochemical potential of electrons $\hat{\mu}_{\text {eon }}(x, y, \omega)$ as a function of $x$ and $y$ in the case where $T=650{ }^{\circ} \mathrm{C}$ and $\tilde{p}_{\mathrm{O}_{2}}=10^{-25.33}$. In the top panels we depict its absolute value $\left|\hat{\mu}_{\text {eon }}\right|$ while at the bottom we show its $\operatorname{argument} \arg \left(\hat{\mu}_{\text {eon }}\right)$. The applied frequency is increased from left to right, going from $0.001 \mathrm{rad} \mathrm{s}^{-1}$ to $1 \mathrm{rad} \mathrm{s}^{-1}$. Only a smart portion of the sample close to the current collector is shown.
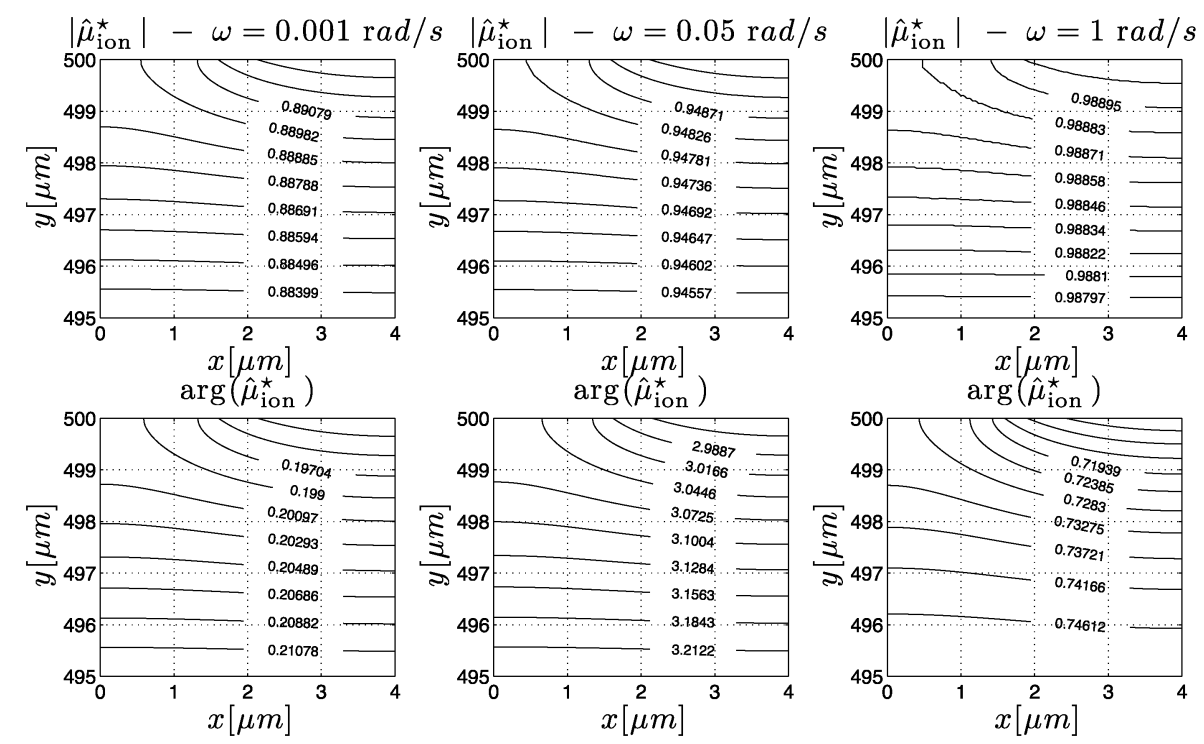

Fig. 6 Similar to Fig. 5, we depict the complex electrochemical potential of ions $\hat{\mu}_{\text {ion }}(x, y, \omega)$ where at the top we show $\left|\hat{\mu}_{\text {ion }}\right|$ and at the bottom $\arg \left(\hat{\mu}_{\text {ion }}\right)$. The conditions are the same as Fig. 5 and so is the frequency range.

We first analyze the qualitative distribution of fluxes: from the gradient of $\left|\hat{\mu}_{\text {eon }}\right|$, which gives an idea of electron flux, we deduce that electrons flow from the gas $\mid$ ceria interface $\Gamma_{5}$ onto the ceria|metal interface $\Gamma_{4}$ through a cross-plane current $\hat{I}_{g}^{\mathrm{CP}}$, and concurrently electrons flow onto the MIEC|metal interface $\Gamma_{5}$ from its mirror symmetric counterpart. Similarly the MIEC|metal interface is vacancy blocking: hereby the vacancies flow from the bottom to the top MIEC|gas interface $\Gamma_{5}$. It is also clear that the complex potential of the electrons $\hat{\mu}_{\text {eon }}$ changes significantly as $\omega$ increases, while $\hat{\mu}_{\text {ion }}$ is relatively unaffected. The penetration depth, which is defined as the vertical displacement from $\Gamma_{4}$ where surface electrons can penetrate into the bulk, decreases with $\omega$ as the $1 \mathrm{D}$ model hints (in eqn (17) the solution decays exponentially with $\left.1 / \sqrt{\tau_{n}^{\star} \omega}\right)$. As $\omega$ increases, the dephasing of $\hat{\mu}_{\text {eon }}$ first increases and then decreases and it is weakly dependent upon the distance from $\Gamma_{4}$, or conversely, the penetration depth into the MIEC. We notice that the same dephasing increases and then decreases for $\hat{\mu}_{\text {ion. }}$. However, while for the vacancies the behavior of $\left|\hat{\mu}_{\text {ion }}\right|$ and $\arg \left(\hat{\mu}_{\text {ion }}\right)$ is qualitatively the same, this is not the case for the electrons, where through a wide array of $\omega$ values, the qualitative behavior of $\left|\hat{\mu}_{\text {eon }}\right|$ and $\arg \left(\hat{\mu}_{\text {eon }}\right)$ is distinctly different.

Deriving the electronic and ionic currents from the computations requires some care and it will not simply be $\nabla\left|\hat{\mu}_{m}\right|$. For example, for electrons, we note that

$$
\hat{\mu}_{\mathrm{eon}}^{(1)}=\left(n^{(1)}-\phi^{(1)}\right) e^{i \omega t} .
$$



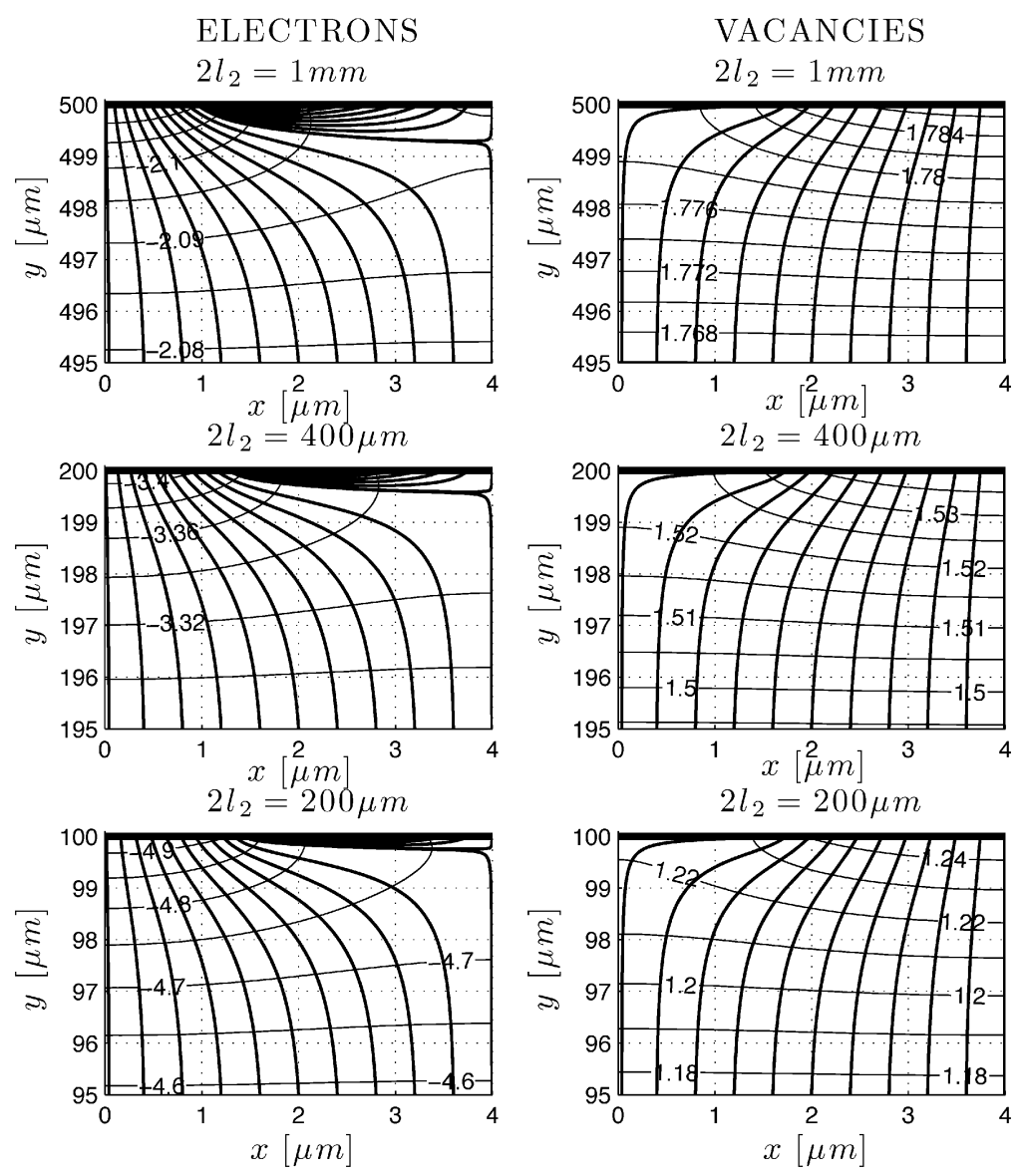

Fig. 7 Potentials and current lines under small bias excitation, i.e., impedance at $\omega=0$, at $T=650{ }^{\circ} \mathrm{C}$ and $\tilde{p}_{\mathrm{O}_{2}}=10^{-25.33}$. The $\hat{\mu}_{\text {eon }}($ left column) and $\hat{\mu}_{v}$ (right column) along with their current lines are plotted. Each row corresponds to a different thickness. Only a small portion of the sample close to the metal current collector is plotted.

We will call the complex electron current $\mathbf{j}_{\mathrm{eon}}^{\mathbb{C}}$, where

$$
\mathbf{j}_{\text {eon }}^{\mathbb{C}}=c_{\text {eon }}^{0} D_{\text {eon }} \mathscr{F}^{-1}\left[\nabla \hat{\mu}_{\text {eon }}^{(1)}\right] .
$$

In the latter $\mathscr{F}^{-1}$ is the inverse of the Fourier transform. The physical current of electrons is the real part of the complex electron current $\ddagger$

$$
\mathbf{j}_{\text {eon }}=\Re\left(\mathbf{j}_{\text {eon }}^{\mathbb{C}}\right) \text {. }
$$

In order to compare the $1 \mathrm{D}$ and $2 \mathrm{D}$ solutions qualitatively, we first focus on the case $\omega=0$ where $\tilde{k}_{f}^{0}=10^{32}$, and we shrink the size of the slab while keeping the same model parameters. This corresponds to a decrease of the aspect ratio of the sample defined as $A R=\frac{l_{2}}{W_{1}+W_{2}}$. We show in Fig. 7 and 8 the results of the computations in the case where the conditions are reducing. As the thickness of the sample $l_{2}$ decreases (from top to bottom row) the area affected by surface reactions thins out; this phenomenon relates to an increase of the polarization resistance. We depict what happens to $R_{\text {ion }}$, $R_{\text {eon }}, R_{\text {ion }}^{\perp}$ and $f_{\text {surf }}$ as $A R$ changes. We notice that decreasing $A R$ corresponds to an increase in effective electronic and ionic resistance compared to the ideal case computed according to Table 3 which in turn corresponds to $A R \rightarrow \infty$. Deviations

$\ddagger$ We remark that for complex valued function $\mu$ in general we have $\operatorname{abs}(\nabla \hat{\mu}) \neq \nabla(\operatorname{abs}(\mu))$.
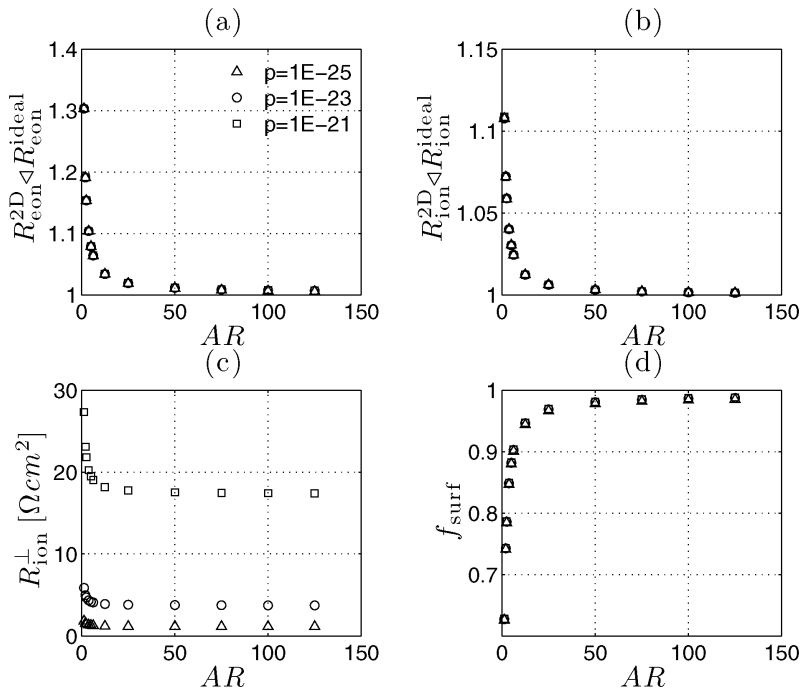

Fig. 8 Deviation of the 2D model from 1D behavior as a function of the aspect ratio $A R=\left(W_{1}+W_{2}\right) / l_{2}$. We consider the case where $\tilde{k}_{f}^{(0)}=10^{32}, T=650{ }^{\circ} \mathrm{C}$ and we set $\tilde{p}_{\mathrm{O}_{2}}=10^{-25.33}(p=$ low $), \tilde{p}_{\mathrm{O}_{2}}=$ $10^{-23.34}(p=\operatorname{med}), \tilde{p}_{\mathrm{O}_{2}}=10^{-20.66}\left(p=\right.$ high). The $R_{\text {eon }}^{2 D}$ and the $R_{\mathrm{ion}}^{2 D}$ monotonically approach their 1D (ideal) value if $A R$ is sufficiently large. $R_{\text {ion }}^{\perp}$ increases while the $f_{\text {surf }}$ decreases with decreasing $A R$, which indicates that if the thickness is reduced enough, the $R_{\mathrm{ion}}^{\perp}$ is not just surface dominated. 
from ideality occur already for $A R \approx 25$, hence even for reasonably large $A R$ the ionic and electronic resistances deviate from the ideal $1 \mathrm{D}$ case - this is clearly shown in Fig. 8a and b. The same applies to the polarization resistance $R_{\text {ion }}^{\perp}$, Fig. 8c, which is flat above $A R \approx 25$, below this value $R_{\text {ion }}^{\perp}$ sharply increases due to bulk polarization effects. As the deviation from the 1D setting starts, not only ionic and electronic resistivities change, but so does the relative importance of surface and drift diffusion effects. Hence the polarization resistance is thickness-dependent, and the dependence is due to the emergence of two-dimensional effects. The increase in drift diffusion resistance due to the motion of electrons from $\Gamma_{5}$ to $\Gamma_{4}$ is also shown in $f_{\text {surf }}$ which approaches unity as $A R \rightarrow \infty$. This effect is even clearer if we plot the electrochemical potentials of electrons and vacancies at $\omega=0$; we note that the affected area shrinks as the sample thickness decreases. And the latter corresponds to an increase of polarization resistance. This effect is purely $2 \mathrm{D}$ and cannot be studied using a 1D model.
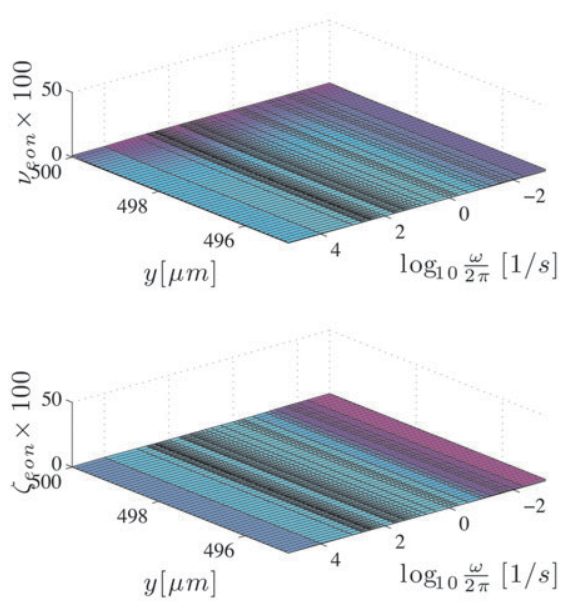

4.3.2 Quantitative analysis. In order to compare the 1D and $2 \mathrm{D}$ solutions quantitatively we define the following two functionals:

$$
\begin{aligned}
\nu\left[\hat{\mu}_{1 D}, \hat{\mu}_{2 D}, \tilde{y}, \omega\right]= & \frac{1}{W_{1}+W_{2}} \\
& \times \frac{\int_{y^{\prime}=\tilde{y}}\left|\hat{\mu}_{1 D}\left(y^{\prime}, \omega\right)-\hat{\mu}_{2 D}\left(\tilde{x}, y^{\prime}, \omega\right)\right| \mathrm{d} \tilde{x}}{\left|\hat{\mu}_{1 D}\left(l_{2}, \omega\right)\right|}, \\
\zeta\left[\hat{\mu}_{1 D}, \hat{\mu}_{2 D}, \tilde{y}, \omega\right]= & \frac{1}{W_{1}+W_{2}} \\
& \times \frac{\left|\int_{y^{\prime}=\tilde{y}} \hat{\mu}_{1 D}\left(y^{\prime}, \omega\right)-\hat{\mu}_{2 D}\left(\tilde{x}, y^{\prime}, \omega\right) \mathrm{d} \tilde{x}\right|}{\left|\hat{\mu}_{1 D}\left(l_{2}, \omega\right)\right|} .
\end{aligned}
$$

The functional $\nu$ describes the "pointwise" distance between $1 \mathrm{D}$ and $2 \mathrm{D}$ solutions of $\hat{\mu}$ at a section $\tilde{y}$ and the functional $\zeta$
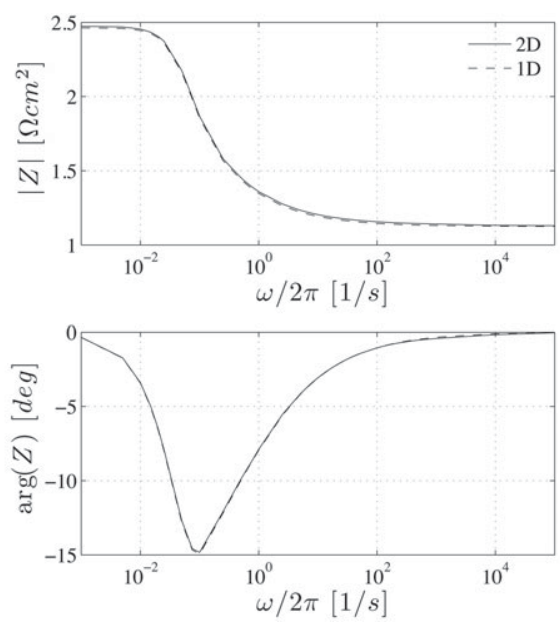

Fig. 9 Plots of the $\nu$ and $\zeta$ values of the electrochemical potential of electrons (only the area near $\Gamma_{4}$ and $\Gamma_{5}$ is shown) as function of $y$ and $\omega$ and of the impedance spectra for $2 l_{2}=1000 \mu \mathrm{m}$ and an aspect ratio $A R=125$. We remark that $\nu$ and $\zeta$ indicate at which frequency the $1 \mathrm{D}$ approximation breaks down.
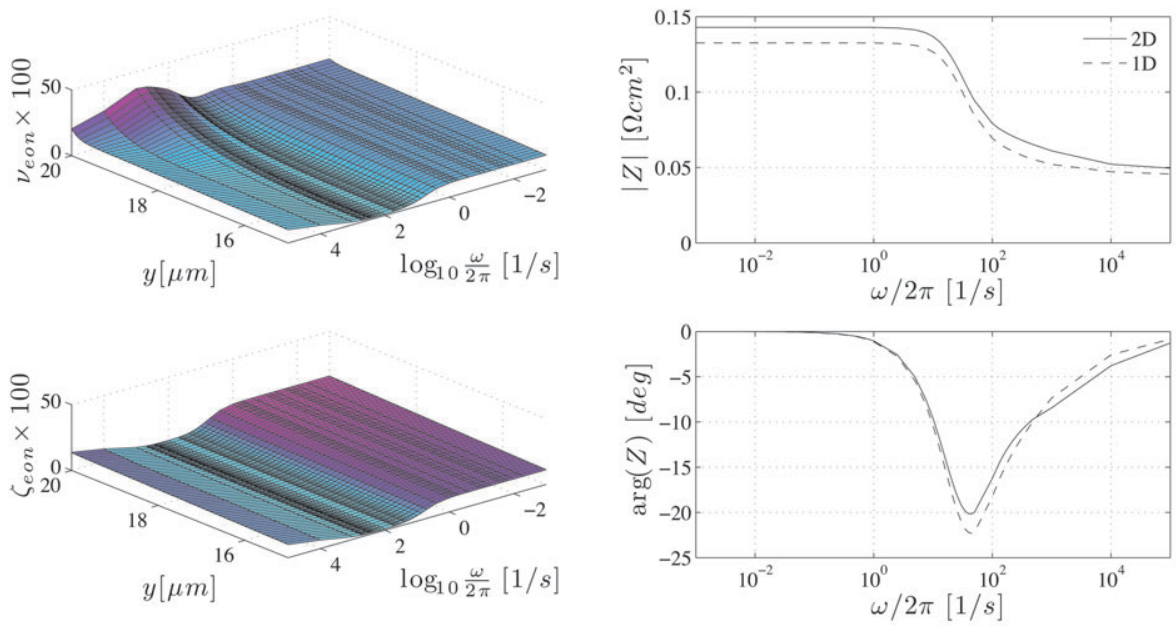

Fig. 10 The same quantities as in Fig. 9 are plotted here. However, $2 l_{2}=40 \mu \mathrm{m}$ which corresponds to $A R=5$ ). A decrease of the aspect ratio induces an increase of both $\nu$ and $\zeta$. The latter means that the (ideal) $1 \mathrm{D}$ impedance and the $2 \mathrm{D}$ impedance spectra are further away as the AR decreases. 
describes the "average" distance between 1D and 2D descriptions. Physically $\nu$ indicates how far apart the 1D and 2D electrochemical potential are, while $\zeta$ "measures" the soundness of fitting a $1 \mathrm{D}$ case with the $2 \mathrm{D}$ model. We can examine the applicability of the 1D approximation for data fitting via $\zeta$.

In order to further compare the 2D model and 1D model and demonstrate the importance of $2 \mathrm{D}$ effects adjacent to the injection sites, the "pointwise" distance $\nu$ and the "average" distance $\zeta$ defined by $(30 \mathrm{~b})$ are computed at the same conditions $\left(T, \tilde{p}_{\mathrm{O}_{2}}, \tilde{k}_{f}^{0}\right)$ in the frequency range of $10^{-3} \leq$ $\omega \leq 10^{5} \mathrm{rad} \mathrm{s}^{-1}$ along the symmetry axis $\Gamma_{2}$, Fig. 9 and 10 . In the first line we plot the case where the sample is thick with respect to the horizontal dimension $(A R=125)$, both the $\nu_{\text {eon }}(\tilde{y}, \omega)=\nu\left[\mu_{\text {eon }, 1 D}, \mu_{\text {eon }, 2 D}, \tilde{y}\right]$ and the $\zeta_{\text {ion }}(\tilde{y}, \omega)=$ $\zeta\left[\mu_{\text {ion }, 1 D}, \mu_{\text {ion }, 2 D}, \tilde{y}\right]$ are extremely small and the adjacency between $1 \mathrm{D}$ and $2 \mathrm{D}$ impedance is near perfect. If we decrease $A R$ to 12.5 , then the $1 \mathrm{D}$ and $2 \mathrm{D}$ solutions tend to be further apart with $\nu_{e} \approx 25 \%$ and $\zeta_{e}$ up to $20 \%$. The difference between the two increases further at $A R=5$ where the difference between impedance spectra is significant.

\section{The effect of diffusivity gradients}

\subsection{Extension of the model}

Interface effects are one of the biggest sources of uncertainty in doped ionics because impurities in doped materials tend to segregate near interfaces and affect electrocatalytic, absorption and diffusivity properties at the same interfaces. Many studies $^{35-37}$ have attempted to address these issues. However, to the authors' knowledge, no continuum model has addressed yet how these changes affect the polarization resistance or the impedance spectra. In this part of the paper we intend to address the effects of nonuniform diffusivities, which are localized near the interfaces, and which we imagine are due to impurity segregation at the exposed surface $\left(\Gamma_{5}\right.$ in Fig. 1) and to the MIEC $\mid$ metal interface $\left(\Gamma_{4}\right)$.

We shall assume that diffusivities near the MIEC|gas interface and MIEC $\mid$ metal interfaces have non-zero derivatives only along the $y$ direction. We further assume that diffusive effects are symmetric on both ends of the sample $y= \pm l_{2}$, and hence do not affect our initial symmetry assumptions. Lastly we suppose that the functional form of the diffusivities are known in the MIEC and are given by

$$
D_{m}^{\star}=1+\left(\frac{D_{m}^{\mathrm{SURF}}}{D_{m}^{\mathrm{BULK}}}-1\right) e^{-\frac{\left|l_{c} \tilde{y} \pm l_{2}\right|}{\lambda_{m}}}
$$

where $m$ can be either eon or ion, and $\lambda_{m}$, the length scale of diffusive changes, is much smaller than $l_{c}$, the characteristic length-scale of the sample $\left(\lambda_{m} \ll l_{c}\right)$. We stress again that the main assumptions are that the diffusivity gradients parallel to the interfaces are null and that the diffusivity gradients do not affect bulk properties of the material nor the defect chemistry. In other words, near-interface effects involve only diffusivities.

If we change the diffusivity of vacancies at the gas $\mid$ ceria $\left(\Gamma_{5}\right)$ and metal|ceria $\left(\Gamma_{4}\right)$ interface by changing $\alpha_{\text {ion }}$, we need to adjust the $\tilde{k}_{f}^{0}$. In order to keep the same rate of injection $\dot{\omega}_{\text {ion }}^{S},(13)$, the $\tilde{k}_{f}^{0}$ satisfies

$$
\tilde{k}_{f}^{(0)}\left(\alpha_{\text {ion }}\right)=\frac{\left(\alpha_{\text {ion }}\right)_{\text {ref }}}{\alpha_{\text {ion }}}\left(\tilde{k}_{f}^{(0)}\right)_{\text {ref }} .
$$

Numerically we use the same approach described for the linear case but we need the error estimator to account for off-diagonal and space dependent parameters-see eqn (51) (in the linear case $a_{11}=a_{22}=1, a_{12}=a_{21}=0$ ).

Finally we note that we assume that the model holds for length-scales just one order of magnitude greater that the lattice parameter. ${ }^{38}$ This approximation can be justified heuristically using the work of Armstrong, ${ }^{39,40}$ which shows that deviations of the continuum drift-diffusion approach from atomistic models are usually small, even in cases where field effects are big.

\subsection{Results for the extended model}

We first ran the model at steady state $(\omega=0)$ with the objective to analyze the $f_{\text {surf }}=\frac{R_{\text {surf }}}{R_{\text {ion }}^{\perp}}$ at $\omega=0$ for a wide array of parameters $\alpha_{\text {eon }}=D_{\text {eon }}^{\mathrm{SUR}} / D_{\text {eon }}^{\mathrm{BULK}}$ and $\alpha_{\text {ion }}=D_{\text {ion }}^{\mathrm{SUR}} / D_{\text {ion }}^{\mathrm{BULK}}$, where $\alpha_{\text {eon }}=\alpha_{\text {ion }}$ and $\lambda_{\text {eon }}=\lambda_{\text {ion }}$ at varying ${\tilde{k_{f}}}_{f}$. For reasonable fitted values (Table 4 ) and for a wide parameter set, we show that the polarization resistance is surface dominated making $f_{\text {surf }} \approx 1$ robustly.

If chemical reaction rates are "sufficiently" slow (e.g. $\tilde{k}_{f}^{0} \approx 10^{32}$ ) and if the sample is sufficiently thick, then the polarization resistance is dominated by surface effects in the linear case $\left(\alpha_{\text {ion }}=1\right)$, corresponding to an absence of diffusive gradients at the exposed surface. If impurities are present at the exposed surface, diffusivities of charged species may change and hence one could argue that the polarization resistance is not surface-dominated. In order to address this point, we ran two limiting cases, one featuring "slow" chemistry $\left(\tilde{k}_{f}^{0}\left(\alpha_{\text {ion }}=1\right) \approx 10^{32}\right)$ and the other one at "fast" chemistry $\left(\tilde{k}_{f}^{0}\left(\alpha_{\text {ion }}=1\right) \approx 10^{34}\right)$. We present the results of these calculations in Fig. 11 where we plot $f_{\text {surf }}$ as a function of both $\alpha_{\text {ion }}=\alpha_{\text {eon }}$ and the diffusive gradients $\lambda_{\text {ion }}=\lambda_{\text {eon }}$. We notice from Fig. 11a that $f_{\text {surf }}$ is close to unity for two orders of magnitude variation of surface-to-bulk diffusivity ratio $0.1 \leq \alpha_{\text {ion }} \leq 10$ and for a wide span of diffusivity length-scales $5 \mathrm{~nm} \leq \lambda_{\text {ion }} \leq 1 \mu \mathrm{m}$. This indicates that if we perturb the surface diffusivity up to one order of magnitude higher or lower than its bulk value its impact on polarization resistance is minimal. The qualitative effect on the impedance is also small, as shown in the four panels of Fig. 12.

If we choose a "fast" chemistry condition instead, e.g. $\tilde{k}_{f}^{0} \approx$ $10^{34}$, the situation changes significantly from the base case $\left(\alpha_{\text {ion }}=1\right)$, Fig. 12b. In this figure we focus on points $\mathrm{A}$ through D. Point A, having $\alpha_{\text {ion }}=0.1$ and $\lambda_{\text {ion }}=5 \mathrm{~nm}$, indicates that near-surface diffusivities are an order of magnitude lower than their bulk value and this deviation is concentrated near the surface: in this case the polarization resistance is drift-diffusion dominated. If the diffusive length scale is increased to $\lambda_{\text {ion }}=1 \mu \mathrm{m}$, while keeping $\alpha_{\text {ion }}=0.1$ (point $\mathrm{B}$ ), the $f_{\text {surf }}$ will not decrease much further. Starting from point $\mathrm{A}$ we can move to point $\mathrm{C}$, where diffusivity 


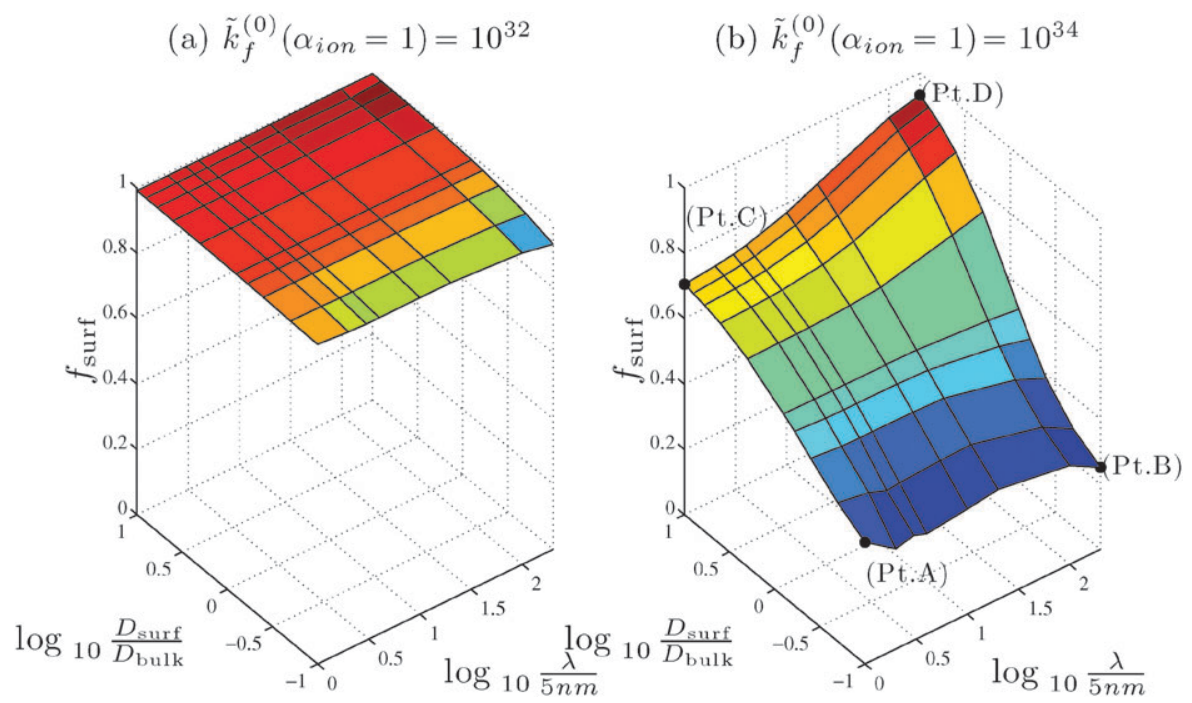

Fig. 11 Depiction of $f_{\text {surf }}$ in the case $T=650{ }^{\circ} \mathrm{C}$ and $\tilde{p}_{\mathrm{O}_{2}}=10^{-25.33}$ as a function of the ratio between near interface and bulk diffusivity, $\alpha_{\text {ion }}=$ $D_{\text {ion }}^{\mathrm{SURF}} / D_{\text {ion }}^{\mathrm{BULK}}$ and $\alpha_{\mathrm{eon}}=D_{\mathrm{eon}}^{\mathrm{SURF}} / D_{\mathrm{eon}}^{\mathrm{BULK}}\left(\alpha_{\text {ion }}=\alpha_{\text {eon }}\right)$, and length scale of the diffusive gradient $\lambda_{\text {ion }}=\lambda_{\text {eon }}$, for $k_{f}^{(0)}=10^{32}(\mathrm{a})$ and $k_{f}^{(0)}=10^{34}(\mathrm{~b})$.
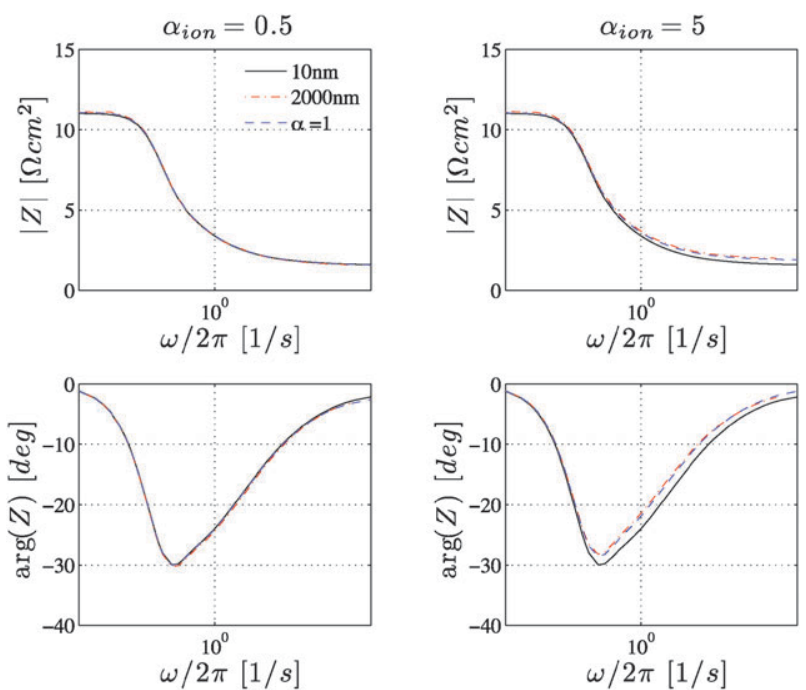

Fig. 12 Impedance of the sample under the conditions: $\tilde{k}_{f}^{(0)}=10^{32}$, $\tilde{p}_{\mathrm{O}_{2}}=10^{-25.33}$ and $T=650^{\circ} \mathrm{C}$, where $\alpha_{\text {eon }}=\alpha_{\text {ion }}\left(\alpha_{m}=D_{m}^{\mathrm{SURF}} / D_{m}^{\mathrm{BULK}}\right)$ and $\lambda_{\text {ion }}=\lambda_{\text {eon }}$. The dashed line represents the case where $\alpha_{\text {ion }}=1$, the triangles and the solid and the dash dotted lines indicate respectively $\lambda_{\text {ion }}=5 \mathrm{~nm}$ and $\lambda_{\text {ion }}=1 \mu \mathrm{m}$. Each column corresponds to a different value of $\alpha_{\text {ion }}$. Only small deviations occur from the case $\alpha_{\text {ion }}=1$.

gradients are sharp $\left(\lambda_{\text {ion }}=5 \mathrm{~nm}\right)$ but the diffusivities at the surface are an order of magnitude greater than their bulk

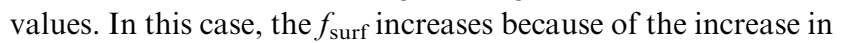
the bulk diffusivity. Going to point $\mathrm{C}$ and then to point $\mathrm{D}$ increases the length-scale of the diffusive effects leading in turn to bigger increase of $f_{\text {surf }}$.

We can summarize our findings as follows:

1. if the rate of injection of electrons is sufficiently "small" (slow chemistry) and of the order of the fitted values reported in Table 4, then the diffusivity grandients localized at interfaces will only affect the polarization resistance and the impedance spectra a little;
2. if the chemistry is sufficiently fast, sharp changes in diffusivity can affect strongly not only the impedance behavior but also the polarization, in particular if the diffusivities increase sufficiently (strictly near the interfaces), the polarization effects will shift to be surface dominated, while a decrease is associated to drift-diffusion dominated polarization resistance.

It is also important to stress that the model was run for a variety of cases such that $\alpha_{\text {eon }} \neq \alpha_{\text {ion }}$ and the same qualitative results mentioned above were recovered.

\section{Concluding remarks}

A general two-dimensional numerical framework has been developed for the coupled surface chemistry, electrochemistry and transport processes in mixed conductors based on the finite element method. As a specialized application of the framework, a time-dependent model was formulated based on basic non-equilibrium thermodynamics for the AC impedance spectra (IS) of a samaria doped ceria (SDC) electrolyte with symmetric metal patterns on both sides, and the IS was simulated for typical fuel cell operation conditions in a uniform gas atmosphere $\left(\mathrm{H}_{2}, \mathrm{H}_{2} \mathrm{O}, \mathrm{Ar}\right)$ at thermodynamic equilibrium using the small perturbation technique.

The validity of the model is demonstrated by fitting to experimental (1D) impedance spectra data of an SDC cell in literature, varying only the reaction rate at the SDC/gas interface. Excellent agreement $(\leq 2 \%$ error) was obtained. We then numerically investigated the influence of the variation of several parameters on the polarization resistance and the impedance spectra, especially within regimes not probable for the 1D studies. Our calculation shows that the 2D effect of cell thickness variation on the spectra becomes pronounced as the aspect ratio goes below a certain threshold ( 25 for this work); surface reaction dominates the polarization resistance when the injection rate at the SDC surface exposed to gas is 
sufficiently slow; sharp gradients in diffusion coefficient strongly influence both impedance behavior and polarization when surface chemistry is sufficiently fast.

The discussions in this work provide useful insights into the correlation between materials properties of SDC and its applications in fuel cells, intensely studied by the solid oxide fuel cell researchers. In addition, the geometric capability (up to 3D) and high computational efficiency makes this numerical framework an ideal tool for the general study of mixed conductors.

\section{Appendix A. Derivation of the impedance equations}

Non-dimensionalization of the eqn (8) with respect to their relevant parameters proves to be crucial in order to understand appropriate time and length scales. We apply the transformations: $(\mathbf{x}, t) \rightarrow(\tilde{\mathbf{x}}, \tilde{t})$ such that $\mathbf{x}=l_{c} \tilde{\mathbf{x}}$ and $t=\tau \tilde{t}$. At this point we suppose the diffusivities $D_{\text {eon }}$ and $D_{\text {ion }}$ are uniform (we shall relax this approximation later). Also, we define $U_{T}=k_{b} T / e, \tilde{\phi}=\phi / U_{T}, \tau_{n}=l_{c}^{2} / D_{\text {eon }}, \tau_{p}=l_{c}^{2} / D_{\text {ion }}$ and $\tau=\min \left(\tau_{n}, \tau_{p}\right)$. Obviously $\nabla_{x}(\cdot)=\frac{1}{l_{c}} \nabla_{\tilde{x}}(\cdot)$ and $\partial_{t}(\cdot)=\frac{1}{\tau} \partial_{\tilde{t}}(\cdot)$. Hence, eqn (8) becomes

$$
\begin{gathered}
\Delta_{\tilde{x}} \tilde{\phi}=\frac{e l_{c}^{2} B}{\varepsilon U_{T}}\left(1+\frac{c_{\mathrm{eon}}^{(0)}}{B} \frac{c_{\mathrm{eon}}}{c_{\mathrm{eon}}^{(0)}}-2 \frac{c_{\mathrm{ion}}^{(0)}}{B} \frac{c_{\text {ion }}}{c_{\mathrm{ion}}^{(0)}}\right), \\
\frac{\tau_{n}}{\tau} \partial_{\tilde{t}} \frac{c_{\mathrm{eon}}}{c_{\mathrm{eon}}^{(0)}}+\nabla_{\tilde{x}} \cdot\left(-\nabla_{\tilde{x}} \frac{c_{\mathrm{eon}}}{c_{\mathrm{eon}}^{(0)}}+\frac{c_{\mathrm{eon}}}{c_{\mathrm{eon}}^{(0)}} \nabla_{\tilde{x}} \tilde{\phi}\right)=0, \\
\frac{\tau_{p}}{\tau} \partial_{\tilde{t}} \frac{c_{\text {ion }}}{c_{\mathrm{ion}}^{(0)}}-\nabla_{\tilde{x}} \cdot\left(\nabla_{\tilde{x}} \frac{c_{\text {ion }}}{c_{\mathrm{ion}}^{(0)}}+2 \frac{c_{\text {ion }}}{c_{\mathrm{ion}}^{(0)}} \nabla_{\tilde{x}} \tilde{\phi}\right)=0,
\end{gathered}
$$

where $c_{\mathrm{eon}}^{(0)}$ and $c_{\mathrm{ion}}^{(0)}$ are equilibrium values. ${ }^{9}$ We define now the Debye length $\lambda_{D}=\sqrt{\frac{\varepsilon U_{T}}{e B}}$ and the parameter $\lambda=\frac{l_{c}}{\lambda_{D}}$ which compares the Debye length against the characteristic length of the system. We suppose $\lambda \gg 1$, which holds true for highly doped MIECs and sufficiently large characteristic dimensions, and we singularly perturb eqn (33a) in order to obtain $^{41}$

$$
1+\frac{c_{\mathrm{eon}}^{(0)}}{B} \frac{c_{\mathrm{eon}}}{c_{\mathrm{eon}}^{(0)}}-2 \frac{c_{\mathrm{ion}}^{(0)}}{B} \frac{c_{\mathrm{ion}}}{c_{\mathrm{ion}}^{(0)}}=0 .
$$

In view of the latter, we can drop (33a); thus we are left with the eqn (33b), (33c) and (34). We now focus on impedance conditions, i.e. we suppose an off-equilibrium perturbation of the boundary conditions which in turn will slightly affect all unknowns (terms with superscript (1) are much smaller than the terms with superscript (0)), i.e.

$$
\begin{aligned}
\tilde{\phi} & =\tilde{\phi}^{(1)}, \\
c_{\mathrm{eon}} & =c_{\mathrm{eon}}^{(0)}+c_{\mathrm{eon}}^{(1)}=c_{\mathrm{eon}}^{(0)}\left(1+\frac{c_{\mathrm{eon}}^{(1)}}{c_{\mathrm{eon}}^{(0)}}\right), \\
c_{\mathrm{ion}} & =c_{\mathrm{ion}}^{(0)}+c_{\mathrm{ion}}^{(1)}=c_{\mathrm{ion}}^{(0)}\left(1+\frac{c_{\mathrm{ion}}^{(1)}}{c_{\mathrm{ion}}^{(0)}}\right) .
\end{aligned}
$$

We now define $n^{(1)}=\frac{c_{\mathrm{eon}}^{(1)}}{c_{\mathrm{eon}}^{(0)}}$ and $p^{(1)}=\frac{c_{\mathrm{ion}}^{(1)}}{c_{\mathrm{ion}}^{(0)}}$ and suppose $c_{\mathrm{eon}}^{(0)}, c_{\mathrm{ion}}^{(0)}$ are uniform and $\phi^{(0)}=0$. If we use the latest definitions and the definitions (35) in eqn (33b) and (33c), we will deduce the following two equations

$$
\frac{\tau_{n}}{\tau} \partial_{t}\left(1+n^{(1)}\right)+\nabla_{\tilde{x}} \cdot\left(-\nabla_{\tilde{x}}\left(1+n^{(1)}\right)+\left(1+n^{(1)}\right) \nabla_{\tilde{x}} \hat{\phi}^{(1)}\right)=0,
$$

$\frac{\tau_{p}}{\tau} \partial_{t}\left(1+p^{(1)}\right)-\nabla_{\tilde{x}} \cdot\left(\nabla_{\tilde{x}}\left(1+p^{(1)}\right)+2\left(1+p^{(1)}\right) \nabla_{\tilde{x}} \hat{\phi}^{(1)}\right)=0$.

If we retain in (36) only first order terms, then we will obtain

$$
\begin{gathered}
\frac{\tau_{n}}{\tau} \partial_{\tilde{t}} n^{(1)}-\Delta_{\tilde{x}} n^{(1)}+\Delta_{\tilde{x}} \hat{\phi}^{(1)}=0, \\
\frac{\tau_{p}}{\tau} \partial_{\tilde{t}} p^{(1)}-\Delta_{\tilde{x}} p^{(1)}-2 \Delta_{\tilde{x}} \hat{\phi}^{(1)}=0 .
\end{gathered}
$$

The electroneutrality condition (34) gives at first order that the dimensionless electron and vacancy concentrations satisfy the following expression:

$$
p^{(1)}=\frac{1}{2} \frac{c_{\mathrm{eon}}^{(0)}}{c_{\mathrm{ion}}^{(0)}} n^{(1)}=\frac{1}{2} \frac{\bar{n}}{\bar{p}} n^{(1)} .
$$

Thus, defining

$$
\begin{aligned}
\tau_{n}^{\star} & =\frac{\tau_{n}+\frac{\bar{n}}{4 \bar{p}} \tau_{p}}{1+\frac{\bar{n}}{4 \bar{p}}}, \\
\tau_{\phi}^{\star} & =\frac{\tau_{p}-\tau_{n}}{1+\frac{4 \bar{p}}{\bar{n}}},
\end{aligned}
$$

helps rewrite (37) as:

$$
\begin{aligned}
& \frac{\tau_{n}^{\star}}{\tau} \partial_{\tilde{t}} n^{(1)}-\Delta_{\tilde{x}} n^{(1)}=0, \\
& \frac{\tau_{\phi}^{\star}}{\tau} \partial_{\tilde{t}} n^{(1)}-\Delta_{\tilde{x}} \hat{\phi}^{(1)}=0 .
\end{aligned}
$$

If we Fourier transform (40) and the boundary conditions with respect to $\tilde{t},{ }^{\S}$ we find the following system of equations (where $(\hat{*})$ indicates a Fourier transformed quantity) ${ }^{\uparrow}$ which we call IS equations:

$$
\begin{array}{r}
\omega \tau_{n}^{\star} \tilde{n}^{(1)}-\Delta \tilde{n}^{(1)}=0 \\
i \omega \tau_{\phi}^{\star} \tilde{n}^{(1)}-\Delta \hat{\phi}^{(1)}=0
\end{array}
$$

with the following boundary conditions given in section 3.2:

$$
\begin{cases}\hat{\phi}^{(1)}=0 \& \hat{n}^{(1)}=0 & \text { on } \Gamma_{1} \\ \partial_{\tilde{x}} \hat{\phi}^{(1)}=0 \& \partial_{\tilde{x}} \hat{n}^{(1)}=0 & \text { on } \Gamma_{2} \& \Gamma_{3} \\ \hat{\phi}^{(1)}=1 \& \partial_{\tilde{y}} \hat{n}^{(1)}=-4 \overline{\bar{n}} \partial_{\tilde{y}} \hat{\phi}^{(1)} & \text { on } \Gamma_{4} \\ \partial_{\tilde{y}} \hat{\phi}^{(1)}=\tilde{A}_{\phi} \hat{n}^{(1)} \& \partial_{\tilde{y}} \hat{n}^{(1)}=\tilde{A}_{n} \hat{n}^{(1)} & \text { on } \Gamma_{5}\end{cases}
$$

$\S \mathrm{We}$ choose the unitary Fourier transform $\hat{f}(\omega)=$ $\frac{1}{\sqrt{2 \pi}} \int_{-\infty}^{\infty} f(x) e^{-i \omega x} \mathrm{~d} x$.

- We factored out the Dirac distribution that comes out of Fourier transformation of an exponential. 


\section{A.1 Weak formulation of the impedance equations}

We can recast the (41) and (42) in weak form taking as test functions $m_{\mathrm{Re}}, m_{\mathrm{Im}} \in \mathrm{H}^{1}\left(\Omega \backslash \Gamma_{1}\right), \psi_{\mathrm{Re}}, \psi_{\mathrm{Im}} \in \mathrm{H}^{1}\left(\Omega \backslash \Gamma_{1} \cup \Gamma_{4}\right)^{42}$

$$
\begin{aligned}
& \omega \tau_{n}^{\star} \int_{\Omega} \hat{n}_{\mathrm{Im}}^{(1)} m_{\mathrm{Re}} \mathrm{d} \tilde{A}-\int_{\Omega} \nabla \hat{n}_{\operatorname{Re}}^{(1)} \cdot \nabla m_{\operatorname{Re}} \mathrm{d} \tilde{A}+\int_{\Gamma_{5}} \tilde{A}_{n} \hat{n}_{\operatorname{Re}}^{(1)} m_{R e} \mathrm{~d} \tilde{A} \\
& -4 \frac{\bar{p}}{\bar{n}} \int_{\Gamma_{4}} \partial_{\tilde{y}} \hat{\phi}_{\operatorname{Re}}^{(1)} m_{\operatorname{Re}} \mathrm{d} \tilde{x}=0 \\
& \omega \tau_{n}^{\star} \int_{\Omega} \hat{n}_{\operatorname{Re}}^{(1)} m_{\mathrm{Im}} \mathrm{d} \tilde{A} \int_{\Omega} \nabla \hat{n}_{\mathrm{Im}}^{(1)} \cdot \nabla m_{\mathrm{Im}} \mathrm{d} \tilde{A}-\int_{\Gamma_{5}} \tilde{A}_{n} \hat{n}_{\operatorname{Im}}^{(1)} m_{\mathrm{Im}} \mathrm{d} \tilde{x} \\
& +4 \frac{\bar{p}}{\bar{n}} \int_{\Gamma_{4}} \partial_{\tilde{y}} \hat{\phi}_{\operatorname{Im}}^{(1)} m_{\operatorname{Im}} \mathrm{d} \tilde{x}=0 \\
& \omega \tau_{\phi}^{\star} \int_{\Omega} \hat{n}_{\mathrm{Im}}^{(1)} \psi_{\mathrm{Re}} \mathrm{d} \tilde{A}-\int_{\Omega} \nabla \hat{\phi}_{\mathrm{Re}}^{(1)} \cdot \psi_{\mathrm{Re}} \mathrm{d} \tilde{A}+\int_{\Gamma_{5}} \tilde{A}_{\phi} \hat{n}_{\mathrm{Re}}^{(1)} \psi_{\mathrm{Re}} \mathrm{d} \tilde{x} \\
& =0 \text {, } \\
& \omega \tau_{\phi}^{\star} \int_{\Omega} \hat{n}_{\operatorname{Re}}^{(1)} \psi_{\operatorname{Im}} \mathrm{d} \tilde{A}+\int_{\Omega} \nabla \hat{\phi}_{\mathrm{Im}}^{(1)} \cdot \psi_{\mathrm{Im}} \mathrm{d} \tilde{A}-\int_{\Gamma_{5}} \tilde{A}_{\phi} \hat{n}_{\operatorname{Im}}^{(1)} \psi_{\mathrm{Im}} \mathrm{d} \tilde{x} \\
& =0,
\end{aligned}
$$

with the condition that:

$$
\begin{aligned}
& \hat{\phi}_{\operatorname{Re}}^{(1)}=0 \text { and } \hat{\phi}_{\operatorname{Im}}^{(1)}=0 \text { on } \Gamma_{1} \\
& \hat{n}_{\operatorname{Re}}^{(1)}=0 \text { and } \hat{n}_{\mathrm{Im}}^{(1)}=0 \text { on } \Gamma_{1} \\
& \hat{\phi}_{\operatorname{Re}}^{(1)}=1 \text { and } \hat{\phi}_{\mathrm{Im}}^{(1)}=0 \text { on } \Gamma_{4}
\end{aligned}
$$

It is easy to show that the sum of the eqn (43) is bounded and thus the bilinear form associated to the weak formulation of eqn (10) with (14) is continuous. Furthermore the problem is weakly coercive, hence it admits one unique solution. ${ }^{43}$

\section{A.2 Error estimator}

The numerical method used to solve the weak problem (43) requires that the local residual for $n_{\mathrm{Re}}$, at a triangular element $K$ of the mesh, can be computed as follows: ${ }^{26}$

$$
\begin{aligned}
\eta_{k, n_{\mathrm{Re}}}= & \int_{K}\left|\nabla \cdot\left(a_{11} \nabla \hat{n}_{\mathrm{Re}, h}^{(1)}+a_{12} \nabla \hat{\phi}_{\mathrm{Re}, h}^{(1)}\right)-\omega \tau_{n}^{\star} \hat{n}_{\mathrm{Im}, h}^{(1)}\right| h^{2} \\
& +\left[\left[a_{11} \frac{\partial \hat{n}_{\mathrm{Re}, h}^{(1)}}{\partial \mathbf{n}}+a_{12} \frac{\partial \hat{\phi}_{\mathrm{Re}, h}^{(1)}}{\partial \mathbf{n}}\right]\right]_{K} h_{K}^{1 / 2} \\
& +\int_{\Gamma_{5} \cap K}\left|\tilde{A}_{n, 2} \hat{n}_{\mathrm{Re}, h}^{(1)}-\partial_{\tilde{y}} \hat{n}_{\mathrm{Re}, h}^{(1)}\right| h^{2} \\
& +\int_{\Gamma_{4} \cap K}\left|\partial_{\tilde{y}} \hat{n}_{\mathrm{Re}, h}^{(1)}-4 \frac{\bar{p}}{\bar{n}} \partial_{\tilde{y}} \hat{n}_{\mathrm{Re}, h}^{(1)}\right| h^{2}+\int_{\left(\Gamma_{2} \cup \Gamma_{3}\right) \cap K}\left|\partial_{\tilde{x}} \hat{n}_{\mathrm{Re}, h}^{(1)}\right| h^{2},
\end{aligned}
$$

where $\llbracket a \rrbracket_{K}$ is the jump of the quantity $a$ across the faces of $K$, $h$ is a measure of the size $K$, while $h_{K}$ is the measure of the size of the sides of $K$. Similar residuals can be found for $n_{\mathrm{Im}}^{(1)}, \phi_{\mathrm{Re}}^{(1)}$, $\phi_{\mathrm{Im}}^{(1)}$. Their sum $\sum_{k} r_{k}$ constitutes a reasonable local a posteriori error estimator. $\sum_{k} r_{k}$ is a weakly coercive upper bound for $a\|u\|_{L_{2}}-b\|\nabla u\|_{L_{2}}$ where $a$ and $b$ are constants and
$u=\left(n_{\mathrm{Re}}^{(1)}, n_{\mathrm{Im}}^{(1)}, \phi_{\mathrm{Re}}^{(1)}, \phi_{\mathrm{Im}}^{(1)}\right)$. More information can be found in the first author's PhD thesis. ${ }^{44}$

\section{Appendix B. Derivation of the non-linear impedance spectra equations}

Under the same small perturbation assumptions we used above we can deduce that the equations that describe the impedance spectra behavior of ions and electrons are given by\|

$$
\begin{gathered}
n^{(1)}=\frac{\bar{n}}{\bar{p}} p^{(1)}, \\
\frac{\tau_{n}}{\tau} \partial_{\tilde{t}} n^{(1)}+\nabla_{\tilde{x}} \cdot\left(-D_{\text {eon }}^{\star}\left(\nabla_{\tilde{x}} n^{(1)}-\nabla_{\tilde{x}} \hat{\phi}^{(1)}\right)\right)=0, \\
\frac{\tau_{p}}{\tau} \partial_{\tilde{t}} p^{(1)}+\nabla_{\tilde{x}} \cdot\left(-D_{\text {ion }}^{\star}\left(\nabla_{\tilde{x}} p^{(1)}+2 \nabla_{\tilde{x}} \hat{\phi}^{(1)}\right)\right)=0 .
\end{gathered}
$$

We start with the electroneutral form of the drift-diffusion equations, where we assume that the diffusion coefficients normalized with respect to their bulk value $D_{m}^{\star}=D_{m}^{\mathrm{SURF}} / D_{m}^{\mathrm{BULK}}$ :

$$
\begin{aligned}
& \frac{\tau_{n}}{\tau} \partial_{\tilde{t}} n^{(1)}+\nabla_{\tilde{x}} \cdot\left(-D_{\text {eon }}^{\star}\left(\nabla_{\tilde{x}} n^{(1)}-\nabla_{\tilde{x}} \hat{\phi}^{(1)}\right)\right)=0, \\
& \frac{\bar{n}}{4 \bar{p}} \frac{\tau_{p}}{\tau} \partial_{\tilde{t}} n^{(1)}+\nabla_{\tilde{x}} \cdot\left(-D_{\text {ion }}^{\star}\left(\frac{\bar{n}}{4 \bar{p}} \nabla_{\tilde{x}} n^{(1)}+\nabla_{\tilde{x}} \hat{\phi}^{(1)}\right)\right)=0 .
\end{aligned}
$$

We first sum the eqn (47a) and (47b) and obtain:

$$
\begin{aligned}
& \left(\frac{\tau_{n}}{\tau}+\frac{\bar{n}}{4 \bar{p}} \frac{\tau_{p}}{\tau}\right) \partial_{\tilde{t}} n^{(1)} \\
& +\nabla_{\tilde{x}} \cdot\left(-\left(D_{\text {eon }}^{\star}+\frac{\bar{n}}{4 \bar{p}} D_{\text {ion }}^{\star}\right) \nabla_{\tilde{x}} n^{(1)}-\left(D_{\text {ion }}^{\star}-D_{\text {eon }}^{r}\right) \nabla_{\tilde{x}} \hat{\phi}^{(1)}\right)=0 .
\end{aligned}
$$

Finally we multiply (47b) by $\frac{4 \bar{p}}{\bar{n}}$ and sum to eqn (47a):

$$
\begin{aligned}
& \left(\frac{\tau_{p}}{\tau}-\frac{\tau_{n}}{\tau}\right) \partial_{\tilde{t}} n^{(1)} \\
& +\nabla_{\tilde{x}} \cdot\left(-\left(D_{\text {ion }}^{\star}-D_{\text {eon }}^{\star}\right) \nabla_{\tilde{x}} n^{(1)}-\left(D_{\text {eon }}^{\star}+\frac{4 \bar{p}}{\bar{n}} D_{\text {ion }}^{\star}\right) \nabla_{\tilde{x}} \hat{\phi}^{(1)}\right)=0 .
\end{aligned}
$$

From eqn (48) and (49), equations (50) follow immediately and so do their coefficients given in (51).

This leads to the following set of equations:

$$
\begin{aligned}
& \frac{\tau_{n}^{\star}}{\tau} \partial_{\tilde{t}} n^{(1)}+\nabla_{\tilde{x}} \cdot\left(-a_{11} \nabla_{\tilde{x}} n^{(1)}-a_{12} \nabla_{\tilde{x}} \hat{\phi}^{(1)}\right)=0, \\
& \frac{\tau_{\phi}^{\star}}{\tau} \partial_{\tilde{t}} n^{(1)}+\nabla_{\tilde{x}} \cdot\left(-a_{21} \nabla_{\tilde{x}} n^{(1)}-a_{22} \nabla_{\tilde{x}} \hat{\phi}^{(1)}\right)=0,
\end{aligned}
$$

$\|$ In order to ensure linearity, we assume that $\left|D_{k} n^{(1)} \nabla \tilde{\phi}^{(1)}\right| \ll$ $\left|D_{k} \nabla n^{(1)}\right| \approx\left|D_{k} \nabla \widetilde{\phi}^{(1)}\right|$. 
where

$$
\begin{aligned}
& a_{11}=\frac{D_{\text {eon }}^{\star}+\frac{\bar{n}}{4 \bar{p}} D_{\text {ion }}^{\star}}{1+\frac{\bar{n}}{4 \bar{p}}} ; a_{12}=\frac{D_{\text {ion }}^{\star}-D_{\text {eon }}^{\star}}{1+\frac{\bar{n}}{4 \bar{p}}} ; \\
& a_{21}=\frac{D_{\text {ion }}^{\star}-D_{\text {eon }}^{\star}}{1+\frac{4 \bar{p}}{\bar{n}}} ; a_{22}=\frac{D_{\text {eon }}^{\star}+\frac{4 \bar{p}}{\bar{n}} D_{\text {ion }}^{\star}}{1+\frac{4 \bar{p}}{\bar{n}}} ;
\end{aligned}
$$

The equations in (50) with appropriate boundary conditions, i.e. (14), are quasi-linear. Hence, they can be Fourier transformed. In addition to that, they can be recast in weak form as in (43):

$$
\begin{aligned}
& \omega \tau_{n}^{\star} \int_{\Omega} \hat{n}_{\mathrm{Im}}^{(1)} m_{R e} \mathrm{~d} \tilde{A}-\int_{\Omega} a_{11} \nabla \hat{n}_{\mathrm{Re}}^{(1)} \cdot \nabla m_{\mathrm{Re}} \mathrm{d} \tilde{A} \\
& -\int_{\Omega} a_{12} \nabla \hat{\phi}_{\operatorname{Re}}^{(1)} \cdot \nabla m_{\operatorname{Re}} \mathrm{d} \tilde{A}+\int_{\Gamma_{5}} \tilde{A}_{n, 2} \hat{n}_{\mathrm{Re}}^{(1)} m_{\mathrm{Re}} \mathrm{d} \tilde{A} \\
& -4 \frac{\bar{p}}{\bar{n}} \int_{\Gamma_{5}} \partial_{\tilde{y}} \hat{\phi}_{\operatorname{Re}}^{(1)} m_{\operatorname{Re}} \mathrm{d} \tilde{x}=0, \\
& \omega \tau_{n}^{\star} \int_{\Omega} \hat{n}_{\operatorname{Re}}^{(1)} m_{\mathrm{Im}} \mathrm{d} \tilde{A}+\int_{\Omega} a_{11} \nabla \hat{n}_{\mathrm{Im}}^{(1)} \cdot \nabla m_{\mathrm{Im}} \mathrm{d} \tilde{A} \\
& +\int_{\Omega} a_{12} \nabla \hat{\phi}_{\mathrm{Im}}^{(1)} \cdot \nabla m_{\mathrm{Im}} \mathrm{d} \tilde{A}-\int_{\Gamma_{5}} \tilde{A}_{n, 2} \hat{n}_{\mathrm{Im}}^{(1)} m_{\mathrm{Im}} \mathrm{d} \tilde{x} \\
& +4 \frac{\bar{p}}{\bar{n}} \int_{\Gamma_{4}} \partial_{\tilde{y}} \hat{\phi}_{\operatorname{Im}}^{(1)} m_{\operatorname{Im}} \mathrm{d} \tilde{x}=0, \\
& \omega \tau_{\phi}^{\star} \int_{\Omega} \hat{n}_{\mathrm{Im}}^{(1)} \psi_{\mathrm{Re}} \mathrm{d} \tilde{A}-\int_{\Omega} a_{21} \nabla \hat{n}_{\mathrm{Re}}^{(1)} \cdot \nabla \psi_{\mathrm{Re}} \mathrm{d} \tilde{A} \\
& -\int_{\Omega} a_{22} \nabla \hat{\phi}_{\operatorname{Re}}^{(1)} \cdot \nabla \psi_{\operatorname{Re}} \mathrm{d} \tilde{A}+\int_{\Gamma_{5}} \tilde{A}_{\phi, 2} \hat{n}_{\operatorname{Re}}^{(1)} \psi_{\operatorname{Re}} \mathrm{d} \tilde{x}=0 \\
& \omega \tau_{\phi}^{\star} \int_{\Omega} \hat{n}_{\mathrm{Re}}^{(1)} \psi_{\mathrm{Im}} \mathrm{d} \tilde{A}+\int_{\Omega} a_{21} \nabla \hat{n}_{\mathrm{Im}}^{(1)} \cdot \nabla \psi_{\mathrm{Im}} \mathrm{d} \tilde{A} \\
& +\int_{\Omega} a_{22} \nabla \hat{\phi}_{\mathrm{Im}}^{(1)} \cdot \nabla \psi_{\mathrm{Im}} \mathrm{d} \tilde{A}-\int_{\Gamma_{5}} \tilde{A}_{\phi, 2} \hat{n}_{\mathrm{Im}}^{(1)} \psi_{\mathrm{Im}} \mathrm{d} \tilde{x}=0
\end{aligned}
$$

where

$$
\begin{aligned}
& \tilde{A}_{n, 2}=a_{11} \tilde{A}_{n}+a_{12} \tilde{A}_{\phi}, \\
& \tilde{A}_{\phi, 2}=a_{21} \tilde{A}_{n}+a_{12} \tilde{A}_{\phi} .
\end{aligned}
$$

\section{Nomenclature}

$\left[\mathrm{Sm}_{\mathrm{Ce}}^{\prime}\right]$ Concentration of dopant in the unit cell

$\bar{n} \quad$ Ratio of the equilibrium concentration of electrons and the dopant concentration

$\bar{p} \quad$ Ratio of the equilibrium concentration of vacancies and the dopant concentration

$\Gamma_{1} \quad$ Horizontal midline of the MIEC sample

$\Gamma_{2} \quad$ Symmetry line of the sample (vertical MIEC midline)
$\Gamma_{3} \quad$ Symmetry line of the sample (vertical metal current collector midline)

$\hat{\phi} \quad$ Time Fourier transform of the dimensionless first order perturbation of the electric potential

$\hat{n} \quad$ Time Fourier transform of the dimensionless first order perturbation of the concentration of electrons

$\lambda$ Ratio of the characteristic length scale and the Debye length

$\lambda_{D} \quad$ Debye length

$\lambda_{m} \quad$ Length scale of the decay of the diffusivity of species $m$ from its surface to its bulk value

$\mu_{\text {eon }} \quad$ Chemical potential of electrons

$\mu_{\text {ion }} \quad$ Chemical potential of vacancies

$\mu_{m} \quad$ Chemical potential of species $m$

$\phi \quad$ Electric potential

$\phi^{(1)} \quad$ Dimensionless first order perturbation of the electric potential

$\tau \quad$ Timescale

$\tau_{\phi}^{\star} \quad$ Timescale of the potential-driven diffusion

$\tau_{n} \quad$ Timescale of electron diffusion

$\tau_{n}^{\star} \quad$ Timescale of the concentration-driven diffusion

$\tau_{p} \quad$ Timescale of vacancy diffusion

$\tilde{\mu}_{m} \quad$ Electrochemical potential of species $m$

$\hat{\mu}_{m}^{\star} \quad$ Star-electrochemical potential of species $\mathrm{m}$, defined as the ratio of its electrochemical potential and its integer charge, i.e. $\hat{\mu}_{m}^{\star}=\mu_{m} / z_{m}$

$\tilde{\phi} \quad$ Dimensionless electric potential, defined as the ratio $\phi / U_{T}$

$\tilde{p_{k}} \quad$ Non-dimensional pressure, i.e. pressure divided by one atmosphere

$\tilde{t} \quad$ Dimensionless time variable

$\tilde{x} \quad$ Dimensionless space variable

$\hat{\mu}_{\text {eon }}^{\star} \quad$ Star-electrochemical potential of vacancies

$B \quad$ Background acceptor doping in number of dopant particles per unit volume

$c_{\mathrm{eon}}^{(0)} \quad$ Equilibrium concentration of electrons

$c_{\mathrm{ion}}^{(0)} \quad$ Equilibrium concentration of vacancies

$c_{m} \quad$ Concentration of species $m$

$c_{m}^{(0)} \quad$ Equilibrium concentration of species $m$

$c_{m}^{(1)} \quad$ First order perturbation concentration of species $m$

$D_{m}^{\star} \quad$ Dimensionless diffusivity, defined as the ratio of the diffusivity of species $m$ divided by its bulk value

$D_{m}^{\mathrm{BULK}}$ Diffusivity of species $m$ in the bulk, here we assume it is the measured diffusivity

$D_{m}^{\mathrm{SURF}}$ Diffusivity of species $m$ at the surface

$D_{\text {eon }} \quad$ Diffusivity of the electrons

$D_{\text {ion }} \quad$ Diffusivity of the vacancies

$D_{m} \quad$ Diffusivity of species $m$

eon Electron

$f_{m} \quad$ Chemical activity of species $m$

ion Vacancy

$K_{g} \quad$ Equilibrium constant for gas phase reactions

$K_{r} \quad$ Equilibrium constant for solid equilibrium reactions

$l_{2} \quad$ Sample thickness

$l_{c} \quad$ Characteristic length scale

$n^{(1)} \quad$ Dimensionless first order perturbation concentration of electrons, defined as the ratio $c_{\mathrm{eon}}^{(1)} / c_{\mathrm{eon}}^{(0)}$

$p_{k} \quad$ Pressure 
$U_{T} \quad$ Thermal voltage defined as the ratio of $k_{b} T$ and the elementary electric charge e, i.e. $U_{T}=k_{b} T / e$

$u_{\text {eon }} \quad$ Electronic mobility

$u_{\text {ion }} \quad$ Vacancy mobility

$u_{m} \quad$ Mobility of species $m$

$z_{m} \quad$ Integer charge of species $m$

$\mathbf{j}_{m}^{P} \quad$ Flux of species $m$ per unit area, expressed as number of particles per unit area

$\dot{\omega}_{m} \quad$ Net rate of creation of $m$ per unit volume

$\mathrm{e}^{\prime} \quad$ Electron

$\mathrm{O}_{\mathrm{O}}^{x} \quad$ Oxygen site in the crystal

$\mathrm{V}_{\mathrm{O}}^{\bullet} \quad$ Vacant site in the crystal

\section{Acknowledgements}

The authors gratefully acknowledge financial support for this work by the Office of Naval Research under grant N00014-05-1-0712. The authors also thank Prof. Frédéric Hecht for his valuable insight and support on FreeFem ++ .

\section{References}

1 J. Jamnik and J. Maier, Phys. Chem. Chem. Phys., 2001, 3, 1668-1678.

2 J. Jamnik, Solid State Ionics, 2003, 157, 19-28.

3 D. S. Mebane, Y. Liu and M. Liu, J. Electrochem. Soc., 2007, 154, A421-A426.

4 J. Fleig, J. Electroceram., 2004, 13, 637-644.

5 S. B. Adler, B. T. Henderson, M. A. Wilson, D. M. Taylor and R. E. Richards, Solid State Ionics, 2000, 134, 35-42.

6 J. Macdonald, J. Chem. Phys., 1973, 58, 4982-5001.

7 W. C. Chueh, W. Lai and S. M. Haile, Solid State Ionics, 2008, 179, 1036-1041.

8 W. Lai and S. M. Haile, Phys. Chem. Chem. Phys., 2008, 10, $865-883$.

9 W. Lai and S. Haile, J. Am. Ceram. Soc., 2005, 88, 2979-2997.

10 S. Park, J. M. Vohs and R. J. Gorte, Nature, 2000, 404, 265-267.

11 Catalysis by Ceria and Related Materials, ed. A. Trovarelli, Imperial College Press, London, 2001, vol. 2.

12 S. B. Adler, J. A. Lane and B. C. H. Steele, J. Electrochem. Soc., 1996, 143, 3554-3564.

13 R. Kee, H. Zhu and D. Goodwin, Proc. Combust. Inst., 2005, 30, 2379-2404.

14 F. A. Kröger and H. J. Vink, Solid State Phys., 1956, 3, 307-435.

15 J. Newman and K. E. Thomas-Alyea, Elecrochemical Systems, John Wiley and Sons, Inc., Hoboken, New Jersey, third edn, 2004.
16 S. B. Adler, J. A. Lane and B. C. H. Steele, J. Electrochem. Soc., 1997, 144, 1884-1890.

17 M. L. Liu and J. Winnick, J. Electrochem. Soc., 1997, 144, 1881-1884.

18 M. L. Liu and J. Winnick, Solid State Ionics, 1999, 118, 21.

19 I. Riess, Solid State Ionics, 2003, 157, 1-17.

20 J. Fleig and J. Maier, J. Electrochem. Soc., 1997, 144, L302-L305.

21 J. Fleig, P. Pham, P. Sztulzaft and J. Maier, Solid State Ionics, 1998, 113-115, 739-747.

22 J. Fleig, Z. Phys. Chem. - Int. J. Res. Phys. Chem. Chem. Phys., 2007, 221, 1149-1159.

23 F. Ciucci, W. C. Chueh, S. M. Haile and D. G. Goodwin, Two-Dimensional Electrochemical Model for Mixed Conductors. a Study of Ceria, 2009, http://arxiv.org/abs/0903.3250.

24 H. Chang and G. Jaffé, J. Chem. Phys., 1952, 20, 1071-1077.

$25 \mathrm{~F}$. Hecht and O. Pironneau, FreeFem ++ , Université Pierre et Marie Curie, 2007.

26 S. Brenner and L. Scott, The Mathematical Theory of Finite Element Methods, Springer-Verlag, 1st edn, 2000, vol. 15.

27 M. Mogensen, N. M. Sammes and G. A. Tompsett, Solid State Ionics, 2000, 129, 63-94.

28 T. A. Davis, Assoc. Comput. Mach., Trans. Math. Software, 2004, 30, 196-199.

29 D. Goodwin, SOFC IX, 2005, 2, 699-707.

30 W. G. Bessler, J. Electrochem. Soc., 2007, 154, B1186-B1191.

31 F. Ciucci and D. G. Goodwin, ECS Trans., 2007, 7, 2075-2082.

32 W. Lai, PhD Thesis, California Institute of Technology, 2007.

33 Impedance Spectroscopy: Theory, Experiment, and Applications, ed. E. Barsoukov and J. Macdonald, Wiley and Sons, New York, 2005.

34 N. Draper and H. Smith, Applied Regression Analysis, Wiley-Interscience, third edn, 1998.

35 A. Hauch, S. H. Jensen, J. B. Bilde-Sorensen and M. Mogensen, J. Electrochem. Soc., 2007, 154, A619-A626.

36 V. A. Sadykov, Y. V. Frolova, V. V. Kriventsov, D. I. Kochubei E. M. Moroz, D. A. Zyuzin, Y. V. Potapova, V. S. Muzykantov, V. I. Zaikovskii, E. B. Burgina, H. Borchert, S. N. Trukhan, V. P. Ivanov, S. Neophytides, E. Kemnitz and K. K. Scheurell, Mater. Res. Soc. Symp. Proc., 2005, K3.6, 835.

37 M. F. Wilkes, P. Hayden and A. K. Bhattacharya, Appl. Surf. Sci., 2003, 206, 12-19.

38 Z. Zhan, T.-L. Wen, H. Tu and Z.-Y. Lu, J. Electrochem. Soc., 2001, 148, A427-A432.

39 R. Armstrong and B. Horrocks, Solid State Ionics, 1997, 94, 181-187.

40 B. R. Horrocks and R. D. Armstrong, J. Phys. Chem. B, 1999, 103 11332-11338.

41 P. A. Marchowich, C. A. Ringhofer and C. Schmeiser, Semiconductor Equations, Springer-Verlag, Wien, New York, 1990.

42 R. Adams and J. Fournier, Sobolev Spaces, Academic Press, New York, 2nd edn, 2003.

43 S. Agmon, Lectures on Elliptic Boundary Value Problems, D. Van Nonstrand, Princeton, NJ, 1965.

44 F. Ciucci, PhD Thesis, California Institute of Technology, 2009. 